\title{
Thermochemical Performance Analysis of the Steam Reforming of Methane in a Fixed Bed Membrane Reformer: A Modelling and Simulation Study
}

\author{
João Paulo Fernando de Medeiros (D), Vitória da Fonseca Dias, José Marcelo da Silva and Jornandes Dias da Silva *
}

Citation: de Medeiros, J.P.F.; da Fonseca Dias, V.; da Silva, J.M.; da Silva, J.D. Thermochemical Performance Analysis of the Steam Reforming of Methane in a Fixed Bed Membrane Reformer: A Modelling and Simulation Study. Membranes 2021, 11, 6. https://dx.doi.org/10.3390/ membranes11010006

Received: 27 August 2020 Accepted: 7 December 2020 Published: 23 December 2020

Publisher's Note: MDPI stays neutral with regard to jurisdictional claims in published maps and institutional affiliations.

Copyright: () 2020 by the authors. Licensee MDPI, Basel, Switzerland. This article is an open access article distributed under the terms and conditions of the Creative Commons Attribution (CC BY) license (https: / / creativecommons.org / licenses/by/4.0/).
Laboratory of Environmental and Energetic Technology, Polytechnic School-UPE, Rua Benfica-455, Madalena, Recife, PE 50750-470, Brazil; joaopaulofernandodemedeiros@gmail.com (J.P.F.d.M.); vi.dias11@hotmail.com (V.d.F.D.); josemarcelo@eletronenergy.com.br (J.M.d.S.)

* Correspondence: jornandesdias@poli.br

\begin{abstract}
Pd-based membrane reformers have been substantially studied in the past as a promising reformer to produce high-purity $\mathrm{H}_{2}$ from thermochemical conversion of methane $\left(\mathrm{CH}_{4}\right)$. A variety of research approaches have been taken in the experimental and theoretical fields. The main objective of this work is a theoretical modelling to describe the process variables of the Steam Reforming of Methane (SRM) method on the Pd-based membrane reformer. These process variables describe the specific aims of each equation of the mathematical model characterizing the performance from reformer. The simulated results of the mole fractions of components (MFCs) at the outlet of the Fixed Bed Reformer (FBR) and Packed-Bed Membrane Reformer (PBMR) have been validated. When the $\mathrm{H}_{2} \mathrm{O} / \mathrm{CH}_{4}$ ratio decreases in PBMR, the Endothermic Reaction Temperature (ERT) is notably increased ( $998.32 \mathrm{~K}$ ) at the outlet of the PBMR's reaction zone. On the other hand, when the $\mathrm{H}_{2} \mathrm{O} / \mathrm{CH}_{4}$ ratio increases in PBMR, the ERT is remarkably decreased $(827.83 \mathrm{~K})$ at the outlet of the PBMR's reaction zone. An increase of the spatial velocity $\left(\mathrm{S}_{\mathrm{sp}}\right)$ indicates a reduction in the residence time of reactant molecules inside PBMR and, thus, a decrease of the ERT and conversion of $\mathrm{CH}_{4}$. In contrast, a reduction of the $\mathrm{S}_{\mathrm{sp}}$ shows an increase of the residence time of reactant molecules within PBMR and, therefore, a rise of the ERT and conversion of $\mathrm{CH}_{4}$. An increase of the $\mathrm{H}_{2} \mathrm{O} / \mathrm{CH}_{4}$ ratio raises the conversion rate $(\mathrm{CR})$ of $\mathrm{CH}_{4}$ due to the reduction of the coke content on the catalyst particles. Conversely, a reduction of the $\mathrm{H}_{2} \mathrm{O} / \mathrm{CH}_{4}$ ratio decreases the $\mathrm{CR}$ of $\mathrm{CH}_{4}$ owing to the increase of the coke content on the catalyst particles. Contrary to the $\mathrm{CR}$ of $\mathrm{CH}_{4}$, the consumptionbased yield (CBY) of $\mathrm{H}_{2}$ sharply decreases with the increase of the $\mathrm{H}_{2} \mathrm{O} / \mathrm{CH}_{4}$ ratio. An increase of the ERT raises the thermochemical energy storage efficiency ( $\eta_{\text {tese }}$ ) from $68.96 \%$ (ERT $\left.=1023 \mathrm{~K}\right), 63.21 \%$ $(\mathrm{ERT}=973 \mathrm{~K})$, and $48.12 \%(\mathrm{ERT}=723 \mathrm{~K})$. The chemical energy, sensible heat, and heat loss reached values of $384.96 \mathrm{~W}, 151.68 \mathrm{~W}$, and $249.73 \mathrm{~W}$ at $973 \mathrm{~K}$. The selectivity of $\mathrm{H}_{2}$ presents higher amounts in the gaseous mixture that varies from 60.98 to 73.18 while $\mathrm{CH}_{4}$ showed lower values ranging from 1.41 to 2.06. Our work is limited to the SRM method. In terms of future uses of this method, new works can be undertaken using novel materials (open-cell foams) and the physical-mathematical model (two-dimensional and three-dimensional) to evaluate the concentration polarization inside membrane reactors.
\end{abstract}

Keywords: membrane reformer; physical-mathematical model; steam reforming; Pd-based membrane; hydrogen production

\section{Introduction}

The production of hydrogen $\left(\mathrm{H}_{2}\right)$ can be carried out through different methods such as thermochemical method (heat and chemical reactions to produce $\mathrm{H}_{2}$ ), reforming of hydrocarbons, biomass gasification, coal gasification, electrolytic method, and biological method. Usually, the thermochemical reforming methods are used to study the Thermochemical Energy Storage (TES) technology of $\mathrm{H}_{2}$. The TES of $\mathrm{H}_{2}$ can be produced from reforming 
reactions with high energy involved in chemical reaction. In particular, $\mathrm{H}_{2}$ can be obtained from thermochemical conversion of $\mathrm{CH}_{4}$ by driving endothermic reaction methods as the SRM and/or dry reforming of methane (DRM) [1]. The choice of the SRM or DRM depends of the desired ending product. If $\mathrm{H}_{2}$ is the desired ending product, then the SRM is preferable. $\mathrm{H}_{2}$ can also provide environmental benefits as a promising renewable energy vector which can be used in several industrial processes [2,3]. Technological development efforts to produce renewable energy have been reported since last century. $\mathrm{H}_{2}$ is one of the most important energy vectors in the universe accounting for almost $75 \%$ of the all universe mass. $\mathrm{H}_{2}$ is considered a clean energy source for the future.

Membrane reformers (MRs) can be used to produce $\mathrm{H}_{2}$ and purify it in packed-bed and/or fluidized-bed setups [4,5]. The production of $\mathrm{H}_{2}$ inside MRs is still attracting the interest of researchers and engineers. This article is focused on the thermochemical conversion of $\mathrm{CH}_{4}$ in a Packed-Bed Membrane Reformer (PBMR). In this setup, $\mathrm{H}_{2}$ gas from shell side (reaction system) passes towards a permeation side through a Pd-based dense membrane [6]. The permselectivity properties of a Pd-based dense membrane such as permeability and selectivity are able to enhance the production of $\mathrm{H}_{2}$ from the thermochemical reforming reactions. The Pd-based dense membrane can act as a remover and it facilitates the selective removal of the gaseous molecule of $\mathrm{H}_{2}$. The removal of $\mathrm{H}_{2}$ through Pd-based dense membrane shifts the equilibrium of reforming reactions to the chosen direction according to the Le Chatelier principle [7-9]. PBMRs offer many potential advantages such as enhanced conversion of hydrocarbons, reduced cost, improved yield, and high selectivity.

PBMR is an apparatus which integrates the reaction zone and permeation zone separated by a Pd-based dense membrane on the same physical equipment. The Pd-based dense membrane is a barrier that allows only certain component $\left(\mathrm{H}_{2}\right)$ to pass through it and it acts as separator [10,11]. Among the metals, $\mathrm{Pd}$ and its alloys have been applied for manufacturing $\mathrm{H}_{2}$ separators as a function of their high membrane permeability towards $\mathrm{H}_{2}$. The mathematical modelling from PBMRs is important to design and optimize this type reformer in order to understand its behavior for a given reaction system. The mathematical models of PBMRs (gas-solid system) can be developed using mass, energy and momentum balance equations for the gas phase as well as mass, energy and momentum balance equations to the solid phase $[3,6,12-14]$. The dynamic performance of a state variable inside PBMRs can be investigated from an initial value up to a steady state of this variable. In this work, a Non-isothermal Pseudo-Homogeneous Dynamic (NIPHD) model is used to model the SRM method inside PBMR. The NIPHD model is described by a system of Nonlinear Partial Differential Equations (NPDEs) that couple to a complex kinetic model of the SRM.

NIPHD models can be used to simulate and analyze the SRM method inside PBMR. The application of NIPHD models can be an excellent alternative to predict faster solutions of systems of NPDEs due to the lower computational time $[15,16]$. In this context, the NIPHD model is able to predict the main characteristics of the SRM method's dynamic performance in PBMR [17]. In addition, the system of NPDEs represents a strong tool for facilitating the project, optimization, and PBMR reformer's control [3,18-20]. The numerical solution of the system of NPDEs has been a great challenge due to the numerical stability. Given this, several numerical methodologies have been used to compute numerical solutions [21,22]. The choice of methodology is dependent on the desired accuracy of the stability and robustness of numerical results of the NPDE system to maintain computational efficiency. The NPDE system of this work has been transformed into a simpler Nonlinear Ordinary Differential Equations (NODEs) system using the Coupled Integral Equation Approach (CIEA) $[23,24]$. The NODE system was solved by the Runge-Kutta Gill method as well as the NODE from the permeation zone.

With the purpose of reducing the research cost and project time, mathematical modelling and computer simulation are extensively used to obtain a better understanding of design parameters in reformers. The approach and solution of physical-mathematical models are still a novelty of membrane reformers to obtain sustainable clean $\mathrm{H}_{2}$ and, thus, the 
topic is a very relevant in the literature. In comparison with traditional methodologies such as finite element, finite volume, etc., that have already been used before in the literature, our methodology can provide results faster than traditional methods and, therefore, the novelty of the present work lies in the determination of the solution method. A comparative analysis had been driven to investigate ERT, $\mathrm{CR}$ of $\mathrm{CH}_{4}$, and feed-based yield (FBY) of $\mathrm{H}_{2}$ inside FBR and PBMR. The effects of the $\mathrm{H}_{2} \mathrm{O} / \mathrm{CH}_{4}$ ratio and $\mathrm{S}_{\mathrm{sp}}$ on the ERT were numerically investigated in PBMR. After checking the effects of the $\mathrm{H}_{2} \mathrm{O} / \mathrm{CH}_{4}$ ratio and $\mathrm{S}_{\mathrm{sp}}$ on the ERT, the effects of these parameters were also studied on the $\mathrm{CR}$ of $\mathrm{CH}_{4}$ and $\mathrm{CBY}$ of $\mathrm{H}_{2}$. In addition, the ERT's effect was verified on the $\eta_{\text {tese, }}$, chemical energy, sensible heat, and heat loss. In addition, the selectivity of components $\left(\mathrm{H}_{2}, \mathrm{CO}, \mathrm{CO}_{2}\right.$, and $\left.\mathrm{CH}_{4}\right)$ was computed in PBMR.

\section{Physical-Mathematical Model}

A schematic setup is used to study the SRM method's thermochemical conversion in PBMR according to Figure 1. The simplified setup from Figure 1 involves a heating module (electric furnace), input reagents $\left(\mathrm{CH}_{4}, \mathrm{H}_{2} \mathrm{O}\right)$, Sweep gas $\left(\mathrm{N}_{2}\right)$, reaction zone, permeation zone, and outlet products $\left(\mathrm{CH}_{4}, \mathrm{H}_{2} \mathrm{O}, \mathrm{H}_{2}, \mathrm{CO}\right.$, and $\left.\mathrm{CO}_{2}\right)$. The physical setup of the PBMR is built by two concentric tubes according to Figure 1 . The inner tube consists of a thin palladium (Pd) dense membrane which contains a permeation zone receiving $\mathrm{H}_{2}$ from the reaction zone through the Pd-based dense membrane. The catalyst loading is placed between the tubes in the annular zone, named the fixed-bed.

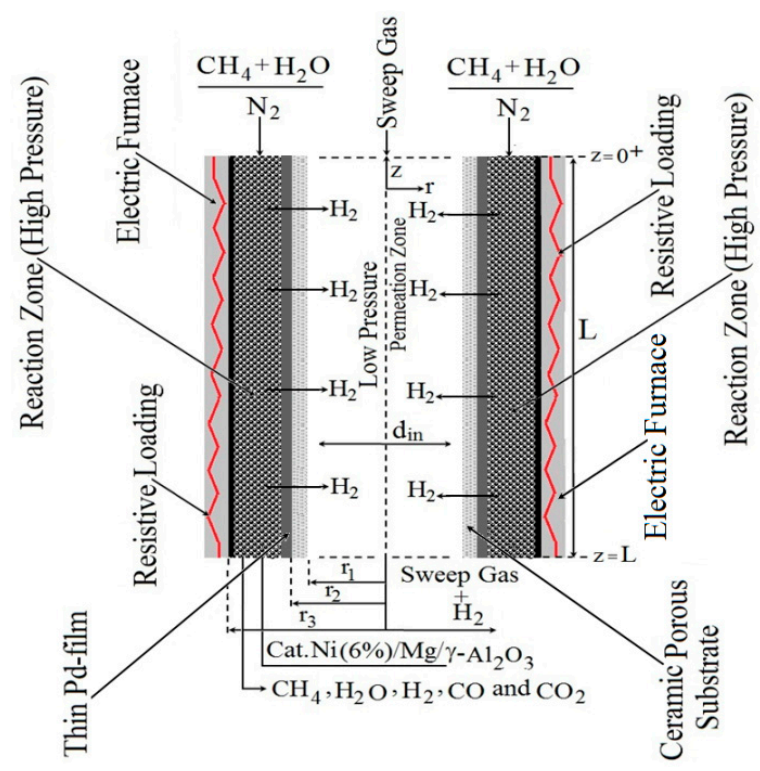

Figure 1. Schematic setup of the physical setup from Packed-Bed Membrane Reformer (PBMR) to study the Steam Reforming of Methane (SRM) method using electric heating.

\subsection{Electric Power of the Electric Furnace}

In Figure 1, a resistive loading inside of an electric furnace has been used to heat the FBMR's reaction zone and therefore the thermal energy storage is used to drive the reforming reactions. The electric power provided by the electric furnace is given for Equation (1) as follows.

$$
\mathrm{P}_{\text {elet. }}=\mathrm{Ri}_{\text {elet. }}^{2}=\mathrm{U}_{\text {ghe }} \mathrm{S}_{\text {she }}\left(\mathrm{T}_{\text {er }}-\mathrm{T}_{\mathrm{g}}\right)
$$


The thermochemical energy storage is obtained by subtracting the product enthalpy (reaction heat) from the reagent enthalpy at room temperature. Thus, the PBMR's chemical energy is obtained using Equation (2) as follows.

$$
\mathrm{Q}_{\text {che }}=\rho_{\mathrm{s}}\left(\frac{1-\varepsilon_{\mathrm{p}}}{\varepsilon_{\mathrm{p}}}\right) \sum_{\mathrm{j}=1}^{3}\left( \pm \Delta \mathrm{H}_{\mathrm{j}}\right) \eta_{\mathrm{j}} \mathrm{R}_{\mathrm{j}}
$$

After cooling the products to the room temperature, the sensible heat of the products (ranging from outer temperature to the room temperature) can be used and therefore the sensible heat can be computed from Equation (3) as follows.

$$
\mathrm{Q}_{\text {sh }}=\sum_{\mathrm{i}=1}^{2} \int_{298 \mathrm{~K}}^{\mathrm{T}_{\mathrm{op}}} \rho_{\mathrm{g}, \mathrm{i}} \mathrm{C}_{\mathrm{p}, \mathrm{g}, \mathrm{i}} \mathrm{F}_{\mathrm{i}} \mathrm{dT} ; \mathrm{i}=\mathrm{H}_{2} \text { and } \mathrm{CO}
$$

From Equations (1)-(3), it is possible to estimate the heat loss using Equation (4) as follows.

$$
\mathrm{Q}_{\text {Loss }}=\mathrm{P}_{\text {elet. }}-\mathrm{Q}_{\text {che }}-\mathrm{Q}_{\text {sh }}
$$

\subsection{Thermochemical Kinetic Model}

The reforming reactions of $\mathrm{CH}_{4}$ are used to produce syngas $\left(\mathrm{H}_{2}\right.$ and $\left.\mathrm{CO}\right)$ and they are highly endothermic [3]. The SRM method has a limited equilibrium and it comprises three major reactions as follows.

$$
\begin{gathered}
\text { SRM : } \mathrm{CH}_{4(\mathrm{~g})}+\mathrm{H}_{2} \mathrm{O}_{(\mathrm{g})} \leftrightarrow \mathrm{CO}_{(\mathrm{g})}+3 \mathrm{H}_{2(\mathrm{~g})} ; \Delta \mathrm{H}_{298 \mathrm{~K}}^{0}=205.8 \mathrm{~kJ} / \mathrm{mol} \\
\text { WGSR : } \mathrm{CO}_{(\mathrm{g})}+\mathrm{H}_{2} \mathrm{O}_{(\mathrm{g})} \leftrightarrow \mathrm{CO}_{2(\mathrm{~g})}+\mathrm{H}_{2(\mathrm{~g})} ; \Delta \mathrm{H}_{298 \mathrm{~K}}^{0}=-41.1 \mathrm{~kJ} / \mathrm{mol} \\
\text { Global SRM : } \mathrm{CH}_{4(\mathrm{~g})}+2 \mathrm{H}_{2} \mathrm{O}_{(\mathrm{g})} \leftrightarrow \mathrm{CO}_{2(\mathrm{~g})}+4 \mathrm{H}_{2(\mathrm{~g})} ; \Delta \mathrm{H}_{298 \mathrm{~K}}^{0}=164.9 \mathrm{~kJ} / \mathrm{mol}
\end{gathered}
$$

The two reforming reactions, Equations (5) and (7), are highly endothermic reactions and they need high temperatures to obtain a high $\mathrm{H}_{2}$ productivity. On the other hand, Equation (6) is a slightly exothermic reaction and it works at low temperature when comparing to Equations (5) and (7).

The global rate equations of the three reactions, Equations (5)-(7), are based on the Langmuir-Hinshelwood kinetic model [3]. The kinetic rates from Equations (5)-(7) are considered more general for nickel (Ni) catalyst and, therefore, the equations of the SRM method are presented as:

$$
\begin{aligned}
\mathrm{R}_{1}= & \frac{\frac{\mathrm{k}_{1}}{\mathrm{P}_{\mathrm{H}_{2}}^{2.5}}\left(\mathrm{P}_{\mathrm{CH}_{4}} \mathrm{P}_{\mathrm{H}_{2} \mathrm{O}}-\frac{\mathrm{P}_{\mathrm{H}_{2}}^{3} \mathrm{P}_{\mathrm{CO}}}{\mathrm{K}_{\mathrm{eq}, 1}}\right)}{(\beta)^{2}} \\
\mathrm{R}_{2}= & \frac{\frac{\mathrm{k}_{2}}{\mathrm{P}_{\mathrm{H}_{2}}}\left(\mathrm{P}_{\mathrm{CO}} \mathrm{P}_{\mathrm{H}_{2} \mathrm{O}}-\frac{\mathrm{P}_{\mathrm{H}_{2}} \mathrm{P}_{\mathrm{CO}_{2}}}{\mathrm{~K}_{\mathrm{eq}, 2}}\right)}{(\beta)^{2}} \\
\mathrm{R}_{3}= & \frac{\frac{\mathrm{k}_{3}}{\mathrm{P}_{\mathrm{H}_{2}}^{3.5}}\left(\mathrm{P}_{\mathrm{CH}_{4}} \mathrm{P}_{\mathrm{H}_{2} \mathrm{O}}-\frac{\mathrm{P}_{\mathrm{H}_{2}}^{4} \mathrm{P}_{\mathrm{CO}_{2}}}{\mathrm{~K}_{\mathrm{eq}, 3}}\right)}{(\beta)^{2}}
\end{aligned}
$$

where $\beta$ is given by Equation (11) as follows.

$$
\beta=1+\frac{\mathrm{F}_{\mathrm{H}_{2} \mathrm{O}, 0}}{\mathrm{~F}_{\mathrm{CH}_{4}, 0}}+\frac{\mathrm{F}_{\mathrm{H}_{2}, 0}}{\mathrm{~F}_{\mathrm{CH}_{4}, 0}}+\mathrm{K}_{\mathrm{CO}} \mathrm{P}_{\mathrm{CO}}+\mathrm{K}_{\mathrm{H}_{2}} \mathrm{P}_{\mathrm{H}_{2}}+\mathrm{K}_{\mathrm{CH}_{4}} \mathrm{P}_{\mathrm{CH}_{4}}+\frac{\mathrm{K}_{\mathrm{H}_{2} \mathrm{O}} \mathrm{P}_{\mathrm{H}_{2} \mathrm{O}}}{\mathrm{P}_{\mathrm{H}_{2}}}
$$


The partial pressures of chemical components $\mathrm{i}, \mathrm{i}=\mathrm{CH}_{4}, \mathrm{H}_{2} \mathrm{O}, \mathrm{CO}, \mathrm{CO}_{2}$ and $\mathrm{H}_{2}$, from Equations (8)-(11) are computed from Equations (12)-(16) below.

$$
\begin{gathered}
\mathrm{P}_{\mathrm{CH}_{4}}=\frac{1-\left(1-\frac{\mathrm{F}_{\mathrm{CH}_{4}}}{\mathrm{~F}_{\mathrm{CH}_{4}, 0}}\right)}{\sigma} \\
\mathrm{P}_{\mathrm{H}_{2} \mathrm{O}}=\frac{\frac{\mathrm{F}_{\mathrm{H}_{2} \mathrm{O}, 0}}{\mathrm{~F}_{\mathrm{FH}_{4}, 0}}-\left(1-\frac{\mathrm{F}_{\mathrm{CH}_{4}}}{\mathrm{~F}_{\mathrm{CH}_{4}, 0}}\right)-\left(\frac{\mathrm{F}_{\mathrm{CO}_{2}}}{\mathrm{~F}_{\mathrm{CH}_{4}, 0}}\right)}{\sigma} \\
\mathrm{P}_{\mathrm{CO}}=\frac{\frac{\mathrm{F}_{\mathrm{CO}, 0}}{\mathrm{~F}_{\mathrm{CH}_{4}, 0}}+\left(1-\frac{\mathrm{F}_{\mathrm{CH}_{4}}}{\mathrm{~F}_{\mathrm{CH}_{4}, 0}}\right)-\left(\frac{\mathrm{F}_{\mathrm{CO}_{2}}}{\mathrm{~F}_{\mathrm{CH}_{4}, 0}}\right)}{\sigma} \\
\mathrm{P}_{\mathrm{H}_{2}}=\frac{\mathrm{F}_{\mathrm{F}_{4}, 0}}{\mathrm{P}_{\mathrm{CO}_{2}}}=\frac{\frac{\mathrm{F}_{\mathrm{CO}_{2,0}, 0}}{\mathrm{~F}_{\mathrm{CH}_{4}, 0}}+\left(\frac{\mathrm{F}_{\mathrm{CO}_{2}}}{\mathrm{~F}_{\mathrm{CH}_{4}, 0}}\right)}{\sigma} \\
\hline
\end{gathered}
$$

where,

$$
\sigma=\frac{1+\sum_{j}^{4} \frac{\mathrm{F}_{\mathrm{i}, 0}}{\mathrm{~F}_{\mathrm{CH}_{4}, 0}}}{\mathrm{P}_{\mathrm{op}}} ; \mathrm{j}=\mathrm{H}_{2} \mathrm{O}, \mathrm{CO}, \mathrm{CO}_{2} \text { and } \mathrm{H}_{2}
$$

The net rates $\left(\mathrm{r}_{\mathrm{i}}\right)$ for each chemical component $\mathrm{i}\left(\mathrm{i}=\mathrm{CH}_{4}, \mathrm{H}_{2} \mathrm{O}, \mathrm{CO}, \mathrm{CO}_{2}\right.$ and $\left.\mathrm{H}_{2}\right)$ are computed through Equation (18) as follows.

$$
r_{i}=\sum_{i=1}^{5} \sum_{j=1}^{3} \eta_{j} \sigma_{i j} R_{j}
$$

From Equation (18), the net rates of each chemical component $i$ are obtained by Equations (19)-(23) as follows.

$$
\begin{aligned}
& r_{C_{4}}=-\eta_{1, \text { av. }} R_{1}-\eta_{3, a v .} R_{3} \\
& r_{\mathrm{H}_{2} \mathrm{O}}=-\eta_{1, \mathrm{av}}, \mathrm{R}_{1}-\eta_{2, \mathrm{av}} \cdot \mathrm{R}_{2}-\eta_{3, \mathrm{av}} \cdot \mathrm{R}_{3} \\
& r_{H_{2}}=3 \eta_{1, a v .} R_{1}+\eta_{2, a v .} R_{2}+4 \eta_{3, a v} R_{3} \\
& r_{C O}=\eta_{1, a v} R_{1}-\eta_{2, a v}, R_{2} \\
& \mathrm{r}_{\mathrm{CO}_{2}}=\eta_{2, \mathrm{av} .} \mathrm{R}_{2}+\eta_{3, \mathrm{av} .} \mathrm{R}_{3}
\end{aligned}
$$

\subsection{PBMR's Mathematical Modelling}

The mathematical modelling inside PBMR's reaction zone is described through the NIPHD model. The development of the NIPHD model takes into account the following assumptions: (1) the NIPHD model is described under non-isothermal conditions inside the reaction zone, (2) the NIPHD model in the reaction zone is plug-flow with axial dispersion under transient condition, (3) the radial dispersion is negligible inside the reaction zone, (4) the gaseous mixture has constant density inside the reaction zone from PBMR, (5) the membrane is considered to be $100 \% \mathrm{H}_{2}$-permselectivity, i.e., the selectivity of $\mathrm{H}_{2}$ is typically very high in dense metallic membranes, (6) the heat exchange between the reaction zone and permeation zone is negligible, (7) the molar flow rates in the reaction zone and permeation zone are constant, (8) the deposition effect of carbon at the surface of catalytic particles has been neglected, (9) the gas behavior in the reaction zone from PBMR was considered as an ideal gas mixture, (10) the bed porosity in the axial direction is considered constant, and (11) chemical reactions are assumed to take place at the surface of catalyst particles. 
These premises are used to build the governing equations of the NIPHD model in PBMR's reaction zone and permeation zone as follows.

\subsubsection{Energy Balance of the Gas Phase in Reaction Zone}

The developed equation provides clear information to drive the temperature distribution of the gas phase in porous medium from PBMR's reaction zone. The energy transport in the gas phase inside the reaction zone is characterized by a balance equation in PBMR's axial direction. Thus, a one-dimensional dynamic equation is modelled for the temperature of the gas phase as follows.

- $\quad$ Energy balance in the gas phase

$$
\rho_{\mathrm{g}, \text { mix. }} C_{\mathrm{p}, \mathrm{g}, \text { mix. }}\left(\frac{\partial \mathrm{T}_{\mathrm{g}}}{\partial \mathrm{t}}+\frac{4 \mathrm{q}_{\mathrm{g}}}{\pi \mathrm{d}_{\mathrm{rz}}^{2}} \frac{\partial \mathrm{T}_{\mathrm{g}}}{\partial \mathrm{z}}\right)=\lambda_{\mathrm{g}, \text { eff }} \frac{\partial^{2} \mathrm{~T}_{\mathrm{g}}}{\partial \mathrm{z}^{2}}-\mathrm{h}_{\mathrm{gs}} \frac{\left(1-\varepsilon_{\mathrm{b}}\right)}{\varepsilon_{\mathrm{b}}} \frac{6}{\mathrm{~d}_{\mathrm{p}}}\left(\mathrm{T}_{\mathrm{g}}-\mathrm{T}_{\mathrm{er}}\right)
$$

The gas phase's effective thermal conductivity is defined as a function of the gaseous mixture thermal conductivity as follows.

$$
\lambda_{\text {g,eff. }}=\varepsilon_{\mathrm{b}} \lambda_{\mathrm{g}, \mathrm{mix}}
$$

where

$$
\lambda_{\text {g,mix. }}=1.52 \times 10^{-11} \mathrm{~T}_{\mathrm{g}, 0}^{3}-4.86 \times 10^{-8} \mathrm{~T}_{\mathrm{g}, 0}^{2}+1.02 \times 10^{-4} \mathrm{~T}_{\mathrm{g}, 0}-3.93 \times 10^{-3}
$$

The suitable initial and boundary conditions from Equations are given as follows.

- $\quad$ Initial condition, i.e., $\mathrm{t}=0$

$$
\left.\mathrm{T}_{\mathrm{g}}\right|_{\mathrm{t}=0,0 \leq \mathrm{z} \leq \mathrm{L}}=0
$$

- At the inlet face surface (upper) of the reaction zone from PBMR, i.e., $z=0^{+}$

$$
\left.\frac{\partial \mathrm{T}_{\mathrm{g}}}{\partial \mathrm{z}}\right|_{\mathrm{z}=0^{+}, \mathrm{t} \geq 0} ^{\mathrm{Up} .}=\frac{\rho_{\mathrm{g}, \text { mix. }} \mathrm{C}_{\mathrm{p}, \mathrm{g}, \text { mix. }}}{\lambda_{\mathrm{g}, \text { mix. }}} \frac{4 \mathrm{q}_{\mathrm{g}}}{\pi \mathrm{d}_{\mathrm{rz}}^{2}}\left(\left.\mathrm{~T}_{\mathrm{g}}\right|_{\mathrm{z}=0^{+}, \mathrm{t} \geq 0} ^{\mathrm{Up} .}-\mathrm{T}_{\mathrm{g}, \infty}^{\mathrm{Up} .}\right)
$$

- $\quad$ At the outlet face surface (bottom) of the reaction zone from PBMR, i.e., $z=L$

$$
\left.\frac{\partial \mathrm{T}_{\mathrm{g}}}{\partial \mathrm{z}}\right|_{\mathrm{z}=\mathrm{L}, \mathrm{t} \geq 0} ^{\text {Bot. }}=\frac{\mathrm{h}_{\mathrm{gs}, \text { eff. }}}{\lambda_{\mathrm{g}, \text { eff. }}}\left(\mathrm{T}_{\mathrm{g}, \infty}^{\text {Bot. }}-\left.\mathrm{T}_{\mathrm{g}}\right|_{\mathrm{z}=\mathrm{L}, \mathrm{t} \geq 0} ^{\text {Bot. }}\right)
$$

\subsubsection{Energy Balance of the Solid Phase in Reaction Zone}

The spherical particle's tortuous structure in the reaction zone could give rise to turbulences with an increase in heat transfer between the solid and gas phases. The thermal energy storage takes place on the solid particles to ensure sufficient energy for processing the endothermic reactions from the SRM method. A promising point is reported by thermal interactions at the surface of catalyst particles where SRM reactions are thermochemically converted. However, the energy balance for the temperature of reforming reactions at the surface of catalytic particles is given as follows.

- Energy balance at the surface of catalytic particles

$$
\rho_{\mathrm{s}} \mathrm{C}_{\mathrm{p}, \mathrm{s}} \frac{\partial \mathrm{T}_{\mathrm{er}}}{\partial \mathrm{t}}=\lambda_{\mathrm{s}, \mathrm{eff}} \frac{\partial^{2} \mathrm{~T}_{\mathrm{er}}}{\partial \mathrm{z}^{2}}+\mathrm{h}_{\mathrm{sg}} \frac{6}{\mathrm{~d}_{\mathrm{p}}} \frac{\left(1-\varepsilon_{\mathrm{b}}\right)}{\varepsilon_{\mathrm{b}}}\left(\mathrm{T}_{\mathrm{g}}-\mathrm{T}_{\mathrm{er}}\right)+\rho_{\mathrm{s}} \frac{\left(1-\varepsilon_{\mathrm{p}}\right)}{\varepsilon_{\mathrm{p}}} \sum_{\mathrm{j}=1}^{3} \pm \Delta \mathrm{H}_{\mathrm{j}} \eta_{\mathrm{j}} \mathrm{R}_{\mathrm{j}}
$$


The solid phase's effective thermal conductivity is defined as a function of the thermal conductivity of the gaseous mixture according to Equation below.

$$
\lambda_{\mathrm{s}, \mathrm{eff}}=\left(1-\varepsilon_{\mathrm{b}}\right) \lambda_{\mathrm{g}, \mathrm{mix}}
$$

The suitable initial and boundary conditions from Equations are given as follows.

- $\quad$ Initial condition at $\mathrm{t}=0$

$$
\left.\mathrm{T}_{\mathrm{er}}\right|_{\mathrm{t}=0,0 \leq \mathrm{z} \leq \mathrm{L}}=\mathrm{T}_{\mathrm{er}, 0}
$$

- $\quad$ At the inlet face surface (upper) of the reaction zone from PBMR, i.e., $z=0^{+}$

$$
\left.\frac{\partial \mathrm{T}_{\mathrm{er}}}{\partial \mathrm{z}}\right|_{\mathrm{z}=0^{+}, \mathrm{t} \geq 0} ^{\mathrm{Up}}=\frac{\mathrm{q}_{\mathrm{h}}}{\lambda_{\mathrm{s}}}
$$

- $\quad$ At the outlet face surface (bottom) of the reaction zone from PBMR, i.e., $z=L$

$$
\left.\frac{\partial \mathrm{T}_{\mathrm{er}}}{\partial \mathrm{z}}\right|_{\mathrm{z}=\mathrm{L}, \mathrm{t} \geq 0} ^{\text {Bot. }}=\frac{\mathrm{h}_{\mathrm{sg}, \text { eff }}}{\lambda_{\mathrm{s}, \mathrm{eff} .}}\left(\mathrm{T}_{\mathrm{er}, \infty}^{\text {Bot. }}-\left.\mathrm{T}_{\mathrm{er}}\right|_{\mathrm{z}=\mathrm{L}, \mathrm{t} \geq 0} ^{\text {Bot. }}\right)
$$

2.3.3. Transport Equations of Chemical Components in Reaction Zone

Based on assumptions mentioned in Section 2.3, chemical components on the reaction zone from PBMR are modelled by Equation (35). Equation (35) reports only the transport equations for chemical components $\mathrm{i}\left(\mathrm{i}=\mathrm{CH}_{4}, \mathrm{H}_{2} \mathrm{O}, \mathrm{CO}\right.$ and $\mathrm{CO}_{2}$ ) without $\mathrm{H}_{2}$ as follows.

- Transport equations of chemical components $\mathrm{i}$ in reaction zone

$$
\frac{\mathrm{u}_{\mathrm{sg}}}{\mathrm{g}} \frac{\partial \mathrm{F}_{\mathrm{i}}}{\partial \mathrm{t}}+\frac{4 \mathrm{q}_{\mathrm{g}}}{\mathrm{S}_{\mathrm{sp}} \pi \mathrm{d}_{\mathrm{rz}}^{2}} \frac{\partial \mathrm{F}_{\mathrm{i}}}{\partial \mathrm{z}}=\frac{\mathrm{D}_{\mathrm{ax}, \mathrm{i}}}{\mathrm{S}_{\mathrm{sp}}} \frac{\partial^{2} \mathrm{~F}_{\mathrm{i}}}{\partial \mathrm{z}^{2}}+\rho_{\mathrm{s}} \mathrm{r}_{\mathrm{rz}}^{2} \mathrm{~L}_{\mathrm{z}}\left(1-\varepsilon_{\mathrm{b}}\right) \mathrm{r}_{\mathrm{i}} ; 0<\mathrm{z}<\mathrm{L}
$$

The suitable initial and boundary conditions from Equations are presented as follows.

- $\quad$ Initial condition, i.e., $\mathrm{t}=0$

$$
\left.\mathrm{F}_{\mathrm{i}}\right|_{\mathrm{t}=0,0 \leq \mathrm{z} \leq \mathrm{L}}=\mathrm{F}_{\mathrm{i}, 0}
$$

- $\quad$ At the inlet face surface (upper) of the reaction zone from PBMR, i.e., $z=0^{+}$

$$
\left.\varepsilon_{\mathrm{b}} \frac{\mathrm{D}_{\mathrm{ax}, \mathrm{i}}}{\mathrm{L}} \frac{\partial \mathrm{F}_{\mathrm{i}}}{\partial \mathrm{z}}\right|_{\mathrm{z}=0^{+}, \mathrm{t} \geq 0} ^{\mathrm{Up} .}=\mathrm{u}_{\mathrm{sg}}\left(\left.\mathrm{F}_{\mathrm{i}}\right|_{\mathrm{z}=0^{+}, \mathrm{t} \geq 0} ^{\mathrm{Up} .}-\mathrm{F}_{\mathrm{i}, \infty}^{\mathrm{Up}}\right)
$$

- $\quad$ At the outlet face surface (bottom) of the reaction zone from PBMR, i.e., $z=L$

$$
\left.\varepsilon_{\mathrm{b}} \frac{\mathrm{D}_{\mathrm{ax}, \mathrm{i}}}{\mathrm{L}} \frac{\partial \mathrm{F}_{\mathrm{i}}}{\partial \mathrm{z}}\right|_{\mathrm{z}=\mathrm{L}, \mathrm{t} \geq 0} ^{\text {Bot. }}=\mathrm{k}_{\mathrm{gs}, \mathrm{eff}}\left(\left.\mathrm{F}_{\mathrm{i}}\right|_{\mathrm{z}=\mathrm{L}, \mathrm{t} \geq 0} ^{\text {Bot. }}-\mathrm{F}_{\mathrm{i}, \infty}^{\text {Bot. }}\right)
$$

As $\mathrm{H}_{2}$ permeates the Pd-based dense membrane, a transport equation is specifically developed for $\mathrm{H}_{2}$ inside the reaction zone. Thus, this equation is reported by Equation (39) as follows.

- Transport equation of $\mathrm{H}_{2}$ in reaction zone

$$
\frac{\mathrm{u}_{\mathrm{sg}}}{\mathrm{g}} \frac{\partial \mathrm{F}_{\mathrm{H}_{2}}}{\partial \mathrm{t}}+\frac{4 \mathrm{q}_{\mathrm{g}}}{\mathrm{S}_{\mathrm{sp}} \pi \mathrm{d}_{\mathrm{rz}}^{2}} \frac{\partial \mathrm{F}_{\mathrm{H}_{2}}}{\partial \mathrm{z}}=\frac{\mathrm{D}_{\mathrm{ax}, \mathrm{H}_{2}}}{\mathrm{~S}_{\mathrm{sp}}} \frac{\partial^{2} \mathrm{~F}_{\mathrm{H}_{2}}}{\partial \mathrm{z}^{2}}+\rho_{\mathrm{s}} \mathrm{r}_{\mathrm{rz}}^{2} \mathrm{~L}_{\mathrm{z}}\left(1-\varepsilon_{\mathrm{b}}\right) \mathrm{r}_{\mathrm{H}_{2}}-\pi \mathrm{d}_{\mathrm{zr}}^{2} \mathrm{~J}_{\mathrm{H}_{2}, \mathrm{per},} ; 0<\mathrm{z}<\mathrm{L}
$$

- $\quad$ Initial condition, i.e., $\mathrm{t}=0$ 


$$
\left.\mathrm{F}_{\mathrm{H}_{2}}\right|_{\mathrm{t}=0,0 \leq \mathrm{z} \leq \mathrm{L}}=\mathrm{F}_{\mathrm{H}_{2}, 0}
$$

- At the inlet face surface (upper) of the reaction zone from PBMR, i.e., $\mathrm{z}=0^{+}$

$$
\left.\varepsilon_{\mathrm{b}} \frac{\mathrm{D}_{\mathrm{ax}, \mathrm{H}_{2}}}{\mathrm{~L}} \frac{\partial \mathrm{F}_{\mathrm{H}_{2}}}{\partial \mathrm{z}}\right|_{\mathrm{z}=0^{+}, \mathrm{t} \geq 0} ^{\mathrm{Up} .}=\mathrm{u}_{\mathrm{sg}}\left(\left.\mathrm{F}_{\mathrm{H}_{2}}\right|_{\mathrm{z}=0^{+}, \mathrm{t} \geq 0} ^{\mathrm{Up} .}-\mathrm{F}_{\mathrm{H}_{2}, \infty}^{\mathrm{Up} .}\right)
$$

- At the outlet face surface (bottom) of the reaction zone from PBMR, i.e., $z=L$

$$
\left.\varepsilon_{\mathrm{b}} \frac{\mathrm{D}_{\mathrm{ax}, \mathrm{H}_{2}}}{\mathrm{~L}} \frac{\partial \mathrm{F}_{\mathrm{H}_{2}}}{\partial \mathrm{z}}\right|_{\mathrm{z}=\mathrm{L}, \mathrm{t} \geq 0} ^{\text {Bot. }}=\mathrm{k}_{\mathrm{gs}, \mathrm{eff}}\left(\left.\mathrm{F}_{\mathrm{H}_{2}}\right|_{\mathrm{z}=\mathrm{L}, \mathrm{t} \geq 0} ^{\text {Bot. }}-\mathrm{F}_{\mathrm{H}_{2}, \infty}^{\text {Bot. }}\right)
$$

\subsubsection{Transport of $\mathrm{H}_{2}$ within the Permeation Zone}

The permeation rate of $\mathrm{H}_{2}$ through the membrane from the high-pressure zone into the permeation zone is assumed to obey the half power pressure law. However, the permeation rate of $\mathrm{H}_{2}$ from the reaction zone into the permeation zone is given as follows.

$$
\mathrm{J}_{\mathrm{H}_{2} \text {,per. }}=\frac{\mathrm{Q}_{0}}{\delta} \exp \left(-\frac{\mathrm{E}_{\mathrm{H}_{2}}}{\mathrm{RT}_{\mathrm{er} .}}\right)\left(\mathrm{P}_{\mathrm{H}_{2, \mathrm{rz}}}^{0.5}-\mathrm{P}_{\mathrm{H}_{2}, \text { per. }}^{0.5}\right)
$$

A differential model allows us to quantify the amount of $\mathrm{H}_{2}$ in the permeation side, but the model has to be consistent with the permeation rate which passes through the Pdbased dense membrane. Thus, a transport equation is developed to estimate the production of $\mathrm{H}_{2}$ in the permeation zone from PBMR as follows.

$$
\frac{\mathrm{dF}_{\mathrm{H}_{2, \text { per. }}}}{\mathrm{dz}}=\frac{\pi \mathrm{d}_{\mathrm{zr}} \mathrm{J}_{\mathrm{H}_{2, \text { per. }}}}{\mathrm{L}} ; 0<\mathrm{z}<\mathrm{L}
$$

- $\quad$ At the inlet face surface (upper) of the permeation zone from PBMR, i.e., $z=0^{+}$

$$
\mathrm{F}_{\mathrm{H}_{2} \text {,per. }} .\left.\right|_{\mathrm{z}=0^{+}} ^{\mathrm{Up} .}=\mathrm{F}_{\mathrm{H}_{2}, \infty}^{\mathrm{Up}}
$$

- At the outlet face surface (bottom) of the permeation zone from PBMR, i.e., $z=L$

$$
\left.\frac{\mathrm{dF}_{\mathrm{H}_{2} \text {,per. }}}{\mathrm{dz}}\right|_{\mathrm{z}=\mathrm{L}} ^{\text {Bot. }}=0
$$

\subsection{Solution of the Mathematical Model}

\subsubsection{Application of the CIEA Method}

The CIEA method can be considered as a powerful technique because of its low computer time relative to traditional methods (finite difference, finite volume, finite element, etc.). The CIEA methodology has been used to transform the NPDE system (Equations (24), (30), (35) and (39)) into an NODE system using the boundary conditions (Equations (28), (29), (33), (34), (37), (38), (41) and (42)) of each NPDE. The coefficients of Equations (47)-(50) can be found in Appendix A of this work. Thus, NODEs (Equations (47)-(50)) are reported as follows.

- Transformed NODE for the gas phase

$$
\begin{aligned}
\alpha_{\mathrm{g}, 1} \frac{\mathrm{d} \overline{\mathrm{T}}_{\mathrm{g}}(\mathrm{t})}{\mathrm{dt}}=\left(\alpha_{\mathrm{g}, 2}-\right. & \left.\lambda_{\mathrm{g}, \text { eff. }} \alpha_{\mathrm{g}, 4}\right) \mathrm{T}_{\mathrm{g}}(0, \mathrm{t})-\left(\alpha_{\mathrm{g}, 2}+\lambda_{\mathrm{g}, \text { eff. }} \alpha_{\mathrm{g}, 5}\right) \mathrm{T}_{\mathrm{g}}(\mathrm{L}, \mathrm{t})-\alpha_{\mathrm{g}, 3} \\
& \left(\overline{\mathrm{T}}_{\mathrm{g}}(\mathrm{t})-\overline{\mathrm{T}}_{\mathrm{s}}(\mathrm{t})\right)+\lambda_{\mathrm{g}, \text { eff. }}\left(\alpha_{\mathrm{g}, 4} \mathrm{~T}_{\mathrm{g}, \infty}^{\mathrm{Up} . \infty}+\alpha_{\mathrm{g}, 5} \mathrm{~T}_{\mathrm{g}, \infty}^{\text {Bot. }}\right)
\end{aligned}
$$

- $\quad$ Transformed NODE for the solid phase 


$$
\begin{aligned}
& \frac{\mathrm{d} \overline{\mathrm{T}}_{\mathrm{er}}(\mathrm{t})=\beta_{\mathrm{s}, 5}\left(\overline{\mathrm{T}}_{\mathrm{g}}(\mathrm{t})-\overline{\mathrm{T}}_{\mathrm{er}}(\mathrm{t})\right)+}{\mathrm{dt}}+\sum_{\mathrm{j}=1}^{3} \pm \Delta \mathrm{H}_{\mathrm{j}} \mathrm{\eta}_{\mathrm{j}} \overline{\mathrm{R}}_{\mathrm{j}}(\mathrm{t})-\beta_{\mathrm{s}, 1} \beta_{\mathrm{s}, 5} \mathrm{~T}_{\mathrm{er}}(\mathrm{L}, \mathrm{t})+\beta_{\mathrm{s}, 1} \\
&\left(\beta_{\mathrm{s}, 5} \mathrm{~T}_{\mathrm{er}, \infty}^{\text {Bot. }}-\beta_{\mathrm{s}, 4}\right)
\end{aligned}
$$

- Transformed NODE for chemical components $\mathrm{i}$ in reaction zone

$$
\begin{aligned}
& \frac{\mathrm{d} \overline{\mathrm{F}}_{\mathrm{i}}(\mathrm{t})}{\mathrm{dt}}=\left(\varphi_{\mathrm{f}, 2} \varphi_{\mathrm{f}, 5}-\varphi_{\mathrm{f}, 1}\right) \mathrm{F}_{\mathrm{i}}(\mathrm{L}, \mathrm{t})-\left(\varphi_{\mathrm{f}, 2} \varphi_{\mathrm{f}, 4}-\varphi_{\mathrm{f}, 1}\right) \mathrm{F}_{\mathrm{i}}(0, \mathrm{t})+\varphi_{\mathrm{f}, 3} \overline{\mathrm{r}}_{\mathrm{i}}(\mathrm{t})+ \\
& \varphi_{\mathrm{f}, 2}\left(\varphi_{\mathrm{f}, 4} \mathrm{~F}_{\mathrm{i}, \infty}^{\mathrm{Up}}-\varphi_{\mathrm{f}, 5} \mathrm{~F}_{\mathrm{i}, \infty}^{\mathrm{Bot}}\right)
\end{aligned}
$$

- Transformed NODE for $\mathrm{H}_{2}$ in reaction zone

$$
\begin{array}{r}
\frac{d \overline{\mathrm{F}}_{\mathrm{H}_{2}}(\mathrm{t})}{\mathrm{dt}}=\left(\vartheta_{\mathrm{H}_{2}, 2} \vartheta_{\mathrm{H}_{2}, 6}-\vartheta_{\mathrm{H}_{2}, 1}\right) \mathrm{F}_{\mathrm{H}_{2}}(\mathrm{~L}, \mathrm{t})-\left(\vartheta_{\mathrm{H}_{2}, 2} \vartheta_{\mathrm{H}_{2}, 5}-\vartheta_{\mathrm{H}_{2}, 1}\right) \mathrm{F}_{\mathrm{H}_{2}}(0, \mathrm{t})+ \\
\vartheta_{\mathrm{H}_{2}, 3} \overline{\mathrm{r}}_{\mathrm{H}_{2}}(\mathrm{t})+\vartheta_{\mathrm{H}_{2}, 2}\left(\vartheta_{\mathrm{H}_{2}, 5} \mathrm{~F}_{\mathrm{H}_{2}, \infty}^{\mathrm{Up}}-\vartheta_{\mathrm{H}_{2}, 6} \mathrm{~F}_{\mathrm{H}_{2}, \infty}^{\text {Bot. }}\right)-\vartheta_{\mathrm{H}_{2}, 4}
\end{array}
$$

\subsubsection{Approximation of the Full Solution}

Several numerical methods have been proposed to solve NPDE systems [15]. The numeric methodology's selection is limited to the desired accuracy on the consistency and robustness of numerical data of the NPDE system. Regarding NODEs, Equations (47)-(50) have been solved by the Runge-Kutta Gill method as well as the NODE in the permeation zone (Equation (44)). On the other hand, the full solution is obtained from Equations (51)-(54) as follows.

- Gas phase's full solution

$$
\mathrm{T}_{\mathrm{g}}(\mathrm{z}, \mathrm{t})=\left.\frac{1}{2} \mathrm{~T}_{\mathrm{g}}(\mathrm{z}, \mathrm{t})\right|_{\mathrm{t}=0}+\sum_{\mathrm{k}=1}^{\infty} \overline{\mathrm{T}}_{\mathrm{g}}\left(\mathrm{t}_{\mathrm{k}}\right) \sin \left(\frac{\mathrm{k} \pi \mathrm{z}}{\mathrm{L}}\right)
$$

- Solid phase's full solution

$$
\mathrm{T}_{\mathrm{er}}(\mathrm{z}, \mathrm{t})=\left.\frac{1}{2} \mathrm{~T}_{\text {er }}(\mathrm{z}, \mathrm{t})\right|_{\mathrm{t}=0}+\sum_{\mathrm{k}=1}^{\infty} \overline{\mathrm{T}}_{\mathrm{er}}\left(\mathrm{t}_{\mathrm{k}}\right) \sin \left(\frac{\mathrm{k} \pi \mathrm{z}}{\mathrm{L}}\right)
$$

- Full solution for chemical components i

$$
\mathrm{F}_{\mathrm{i}}(\mathrm{z}, \mathrm{t})=\left.\frac{1}{2} \mathrm{~F}_{\mathrm{i}}(\mathrm{z}, \mathrm{t})\right|_{\mathrm{t}=0}+\sum_{\mathrm{k}=1}^{\infty} \overline{\mathrm{F}}_{\mathrm{i}}\left(\mathrm{t}_{\mathrm{k}}\right) \sin \left(\frac{\mathrm{k} \pi \mathrm{z}}{\mathrm{L}}\right)
$$

- Full solution for chemical components $\mathrm{H}_{2}$.

$$
\mathrm{F}_{\mathrm{H}_{2}}(\mathrm{z}, \mathrm{t})=\left.\frac{1}{2} \mathrm{~F}_{\mathrm{H}_{2}}(\mathrm{z}, \mathrm{t})\right|_{\mathrm{t}=0}+\sum_{\mathrm{k}=1}^{\infty} \overline{\mathrm{F}}_{\mathrm{H}_{2}}\left(\mathrm{t}_{\mathrm{k}}\right) \sin \left(\frac{\mathrm{k} \pi \mathrm{z}}{\mathrm{L}}\right)
$$

\section{Results}

\subsection{Model Parameters for Simulations}

A physical-mathematical model has been used to investigate the SRM method's thermochemical conversion in PBMR using an external energy loading. A mathematical model is developed to simulate the energy transfer of the gaseous and solid phases and transport of chemical components coupled to the SRM method's thermochemical kinetic model in PBMR. A computational algorithm using the FORTRAN 95 language has been elaborated by the authors to compute the results as in the model equations of this work. In Tables 1 and 2, the geometrical characteristics from PBMR, catalytic bed's properties, and operating conditions at the inlet from PBMR are shown. 
Table 1. PBMR's Geometrical characteristics and the catalytic bed's properties.

\begin{tabular}{lll}
\hline & PBMR & Sources \\
\hline Geometrical characteristics & & \\
$\mathrm{L}(\mathrm{m})$ & 0.18 & Estimated \\
$\mathrm{r}_{\mathrm{rz}}(\mathrm{m})$ & 0.0020 & Ref. [3] \\
$\mathrm{r}_{1}(\mathrm{~m})$ & 0.0010 & Ref. [3] \\
$\mathrm{r}_{2}(\mathrm{~m})$ & 0.0015 & Ref. [3] \\
$\mathrm{r}_{3}(\mathrm{~m})$ & 0.035 & Ref. [3] \\
$\delta_{\mathrm{m}, \mathrm{av} .}(\mu \mathrm{m})$ & 3.17 & Estimated \\
\hline Properties of the catalyst bed & & \\
$\varepsilon_{\mathrm{b}}\left(\mathrm{m}^{3}\right.$ gas $/ \mathrm{m}^{3}$ reformer $)$ & 0.39 & Ref. [3] \\
$\varepsilon_{\mathrm{p}}\left(\mathrm{m}^{3}\right.$ gas $/ \mathrm{m}^{3}$ reformer $)$ & 0.43 & Ref. [3] \\
$\eta_{1}($ Equation $(5))$ & 0.0121 & Ref. [3] \\
$\eta_{2}($ Equation $(6))$ & 0.0169 & Ref. [3] \\
$\eta_{3}($ Equation $(7))$ & 0.0191 & Ref. [3] \\
$\mathrm{d}_{\mathrm{p}}(\mathrm{m})$ & $79 \mu \mathrm{m}$ & Estimated \\
$\rho_{\mathrm{s}}\left(\mathrm{kg} / \mathrm{m}^{3}\right)$ & 2.500 & Ref. [6] \\
$\mathrm{S}_{\mathrm{p}}\left(\mathrm{h}^{-1}\right)$ & 0.796 & Ref. [6] \\
$\mathrm{M}_{\mathrm{bed}}(\mathrm{kg})$ & $9.56 \times 10^{-3}$ & Estimated \\
\hline
\end{tabular}

Table 2. Operating conditions at the inlet from PBMR.

\begin{tabular}{lll}
\hline & PBMR & Sources \\
\hline Operating conditions & & \\
$\mathrm{q}_{\mathrm{g}}\left(\mathrm{m}^{3} / \mathrm{h}\right)$ & $4.473 \times 10^{-6}$ & Ref. [6] \\
$\mathrm{V}_{\mathrm{sg}}(\mathrm{m} / \mathrm{h})$ & $3.34 \times 10^{-2}$ & Ref. [6] \\
$\mathrm{g}\left(\mathrm{m} / \mathrm{h}^{2}\right)$ & $1.271 \times 10^{8}$ & Ref. [6] \\
$\rho_{\mathrm{g}}\left(\mathrm{k} / \mathrm{m}^{3}\right)$ & 0.1692 & Ref. [6] \\
$\mathrm{Q}_{0}\left(\mathrm{kmol} / \mathrm{m} \mathrm{kPa}{ }^{0.5} \mathrm{~h}\right)$ & $7.173 \times 10^{-5}$ & Ref. [6] \\
$\mathrm{E}_{\mathrm{p}}\left(\mathrm{kPa} \mathrm{m} / \mathrm{kmol}^{3}\right)$ & $5.576 \times 10^{4}$ & Ref. [6] \\
$\mathrm{R}(\mathrm{kPa} \mathrm{m} / \mathrm{kmol} \mathrm{K})$ & 8.314 & Ref. [6] \\
$\mathrm{T}_{\mathrm{g}, 0}(\mathrm{~K})$ & 673 & Ref. [6] \\
$\mathrm{T}_{\mathrm{er}, 0}(\mathrm{~K})$ & 673 & Ref. [6] \\
$\mathrm{P}_{\mathrm{op} .}(\mathrm{kPa})$ & 950 & Ref. [3] \\
$\mathrm{P}_{\mathrm{op}, \mathrm{per}}(\mathrm{kPa})$ & 150 & Ref. [3] \\
$\mathrm{F}_{\mathrm{CH}}$, in. $(\mathrm{kmol} \mid \mathrm{h})$ & 0.282 & Ref. [3] \\
$\mathrm{F}_{\mathrm{H}_{2} \mathrm{O}, \text { in. }}(\mathrm{kmol} \mid \mathrm{h})$ & 0.602 & Ref. [3] \\
$\mathrm{F}_{\mathrm{H}_{2}, \text { in. }}(\mathrm{kmol} \mid \mathrm{h})$ & 0.076 & Ref. [3] \\
$\mathrm{F}_{\mathrm{CO}, \text { in. }}(\mathrm{kmol} \mid \mathrm{h})$ & 0.032 & Ref. [3] \\
$\mathrm{F}_{\mathrm{CO}}$, in. $(\mathrm{kmol} \mid \mathrm{h})$ & $6.32 \times 10^{-5}$ & Ref. [3] \\
$\mathrm{F}_{\mathrm{sweep}}(\mathrm{kmol} \mid \mathrm{h})$ & $5 \mathrm{~F}_{\mathrm{CH}}$, in. & Ref. [3] \\
\hline
\end{tabular}

In order to ensure good results of the SRM method on PBMR, the convergence criterion for all results of this work has been secured using the ratio between the new variable value and the old variable value according to the new variable value as follows.

$$
\left|\frac{\alpha_{\text {new }, \mathrm{i}}^{\mathrm{k}+1}-\alpha_{\text {old,i }}^{\mathrm{k}}}{\alpha_{\text {new,i }}^{\mathrm{k}+1}}\right| \leq 10^{-6}
$$

After specifying the main geometrical characteristics from PBMR, the catalytic bed's properties and operating conditions at the inlet from PBMR are shown in the above tables. Tables 3 and 4 show the numerical values of kinetic constants, equilibrium adsorption constants, equilibrium constants, thermophysical parameters, and dispersion coefficients of chemical components $i$ in the reaction zone from PBMR. The parameter values of Tables 1-4 are used to feed the developed computational algorithm for this work. 
Table 3. Numeric values of kinetic constants, adsorption constants, and equilibrium constants for simulating of the SRM method on PBMR.

\begin{tabular}{lll}
\hline & Model Parameters & Sources \\
\hline Kinetic constants & & \\
$\mathrm{k}_{1}\left(\mathrm{kmol} \mathrm{kPa}^{0.5} / \mathrm{kg}_{\text {cat. }} \mathrm{h}\right)$ & $7.215 \times 10^{14}$ & Ref. [3] \\
$\mathrm{k}_{2}\left(\mathrm{kmol} \mathrm{kPa}^{-1} / \mathrm{kg}_{\text {cat. }} \mathrm{h}\right)$ & $1.218 \times 10^{3}$ & Ref. [3] \\
$\mathrm{k}_{3}\left(\mathrm{kmol} \mathrm{kPa}^{0.5} / \mathrm{kg}_{\text {cat. }} \mathrm{h}\right)$ & $9.701 \times 10^{15}$ & Ref. [3] \\
\hline Equilibrium adsorption constants & & \\
$\mathrm{K}_{\mathrm{CH}}\left(\mathrm{kPa}^{-1}\right)$ & $8.974 \times 10^{-7}$ & Ref. [3] \\
$\mathrm{K}_{\mathrm{H}_{2} \mathrm{O}}(-)$ & $3.701 \times 10^{4}$ & Ref. [3] \\
$\mathrm{K}_{\mathrm{H}_{2}}\left(\mathrm{kPa}^{-1}\right)$ & $8.987 \times 10^{-12}$ & Ref. [3] \\
$\mathrm{K}_{\mathrm{CO}}\left(\mathrm{kPa}^{-1}\right)$ & $5.671 \times 10^{-6}$ & Ref. [3] \\
\hline Equilibrium constants & & \\
$\mathrm{K}_{\text {eq, }, 1}\left(\mathrm{kPa}^{2}\right)$ & $2.135 \times 10^{7}$ & Ref. [3] \\
$\mathrm{K}_{\text {eq, } 2}(-)$ & 13.015 & Ref. [3] \\
$\mathrm{K}_{\text {eq, }, 3}\left(\mathrm{kPa}^{2}\right)$ & $7.102 \times 10^{5}$ & Ref. [3] \\
\hline
\end{tabular}

Table 4. Numerical values of thermophysical parameters and dispersion coefficients of chemical components for simulating the SRM method in PBMR.

\begin{tabular}{|c|c|c|}
\hline & Model Parameters & Sources \\
\hline \multicolumn{3}{|c|}{ Thermophysical parameters } \\
\hline $\mathrm{C}_{\mathrm{p}, \mathrm{g}, \mathrm{i}}(\mathrm{J} / \mathrm{kg} \mathrm{K})$ & 0.987 & Ref. [6] \\
\hline$\lambda_{\mathrm{g}, \mathrm{eff}}(\mathrm{W} / \mathrm{m} \mathrm{K})$ & 0.0185 & Equation (25) \\
\hline$h_{g s}\left(W / m^{2} K\right)$ & 1.902 & Ref. [6] \\
\hline $\mathrm{C}_{\mathrm{p}, \mathrm{s}}(\mathrm{J} / \mathrm{kg} \mathrm{K})$ & 336 & Ref. [6] \\
\hline$\lambda_{\mathrm{s}, \mathrm{eff}}(\mathrm{W} / \mathrm{m} \mathrm{K})$ & 0.0289 & Equation (31) \\
\hline$\Delta \mathrm{H}_{\left(700^{\circ} \mathrm{C}\right)}^{\text {Eq.(5) }}(\mathrm{kJ} / \mathrm{kmol})$ & 281.83 & Ref. [6] \\
\hline$\Delta \mathrm{H}_{\left(700^{\circ} \mathrm{C}\right)}^{\mathrm{Eq} \cdot(6)}(\mathrm{kJ} / \mathrm{kmol})$ & -35.67 & Ref. [6] \\
\hline$\Delta \mathrm{H}_{\left(700^{\circ} \mathrm{C}\right)}^{\mathrm{Eq} \cdot(7)}(\mathrm{kJ} / \mathrm{kmol})$ & 209.51 & Ref. [6] \\
\hline \multicolumn{3}{|c|}{ Dispersion coeffs. of components i } \\
\hline $\mathrm{D}_{\mathrm{ax}, \mathrm{CH}_{4}}\left(\mathrm{~m}^{2} / \mathrm{h}\right)$ & 0.0289 & Ref. [6] \\
\hline $\mathrm{D}_{\mathrm{ax}, \mathrm{H}_{2} \mathrm{O}}\left(\mathrm{m}^{2} / \mathrm{h}\right)$ & 0.0379 & Ref. [6] \\
\hline $\mathrm{D}_{\mathrm{ax}, \mathrm{H}_{2}}\left(\mathrm{~m}^{2} / \mathrm{h}\right)$ & 0.0201 & Ref. [6] \\
\hline $\mathrm{D}_{\mathrm{ax}, \mathrm{CO}}\left(\mathrm{m}^{2} / \mathrm{h}\right)$ & 0.0341 & Ref. [6] \\
\hline $\mathrm{D}_{\mathrm{ax}, \mathrm{CO}_{2}}\left(\mathrm{~m}^{2} / \mathrm{h}\right)$ & 0.0189 & Ref. [6] \\
\hline
\end{tabular}

\subsection{Validation by Comparison against Published Data}

Mole Fractions at the Outlet of FBR and PBMR

To ensure the validity of the proposed model, authors have made a comparison of simulated results (SRs) against published data in the open literature. The SRs are computed from a developed computer code by authors. Figure 2 compares the literature results and simulating results of MFCs $i$ at the outlet of the FBR and PBMR. Equation (56) has been used to compute the MFCs i values at the outlet of the reaction zone from FBR and PBMR.

$$
\text { MFCs } s_{i, \text { out. }} \text { of FBR and FBMR }=\frac{\mathrm{RT}_{\text {op. }}}{\mathrm{q}_{\mathrm{g}} \mathrm{P}_{\mathrm{op}}} \mathrm{F}_{\mathrm{i}} ; \mathrm{i}=\mathrm{CH}_{4}, \mathrm{H}_{2} \mathrm{O}, \mathrm{H}_{2}, \mathrm{CO} \text { and } \mathrm{CO}_{2}
$$



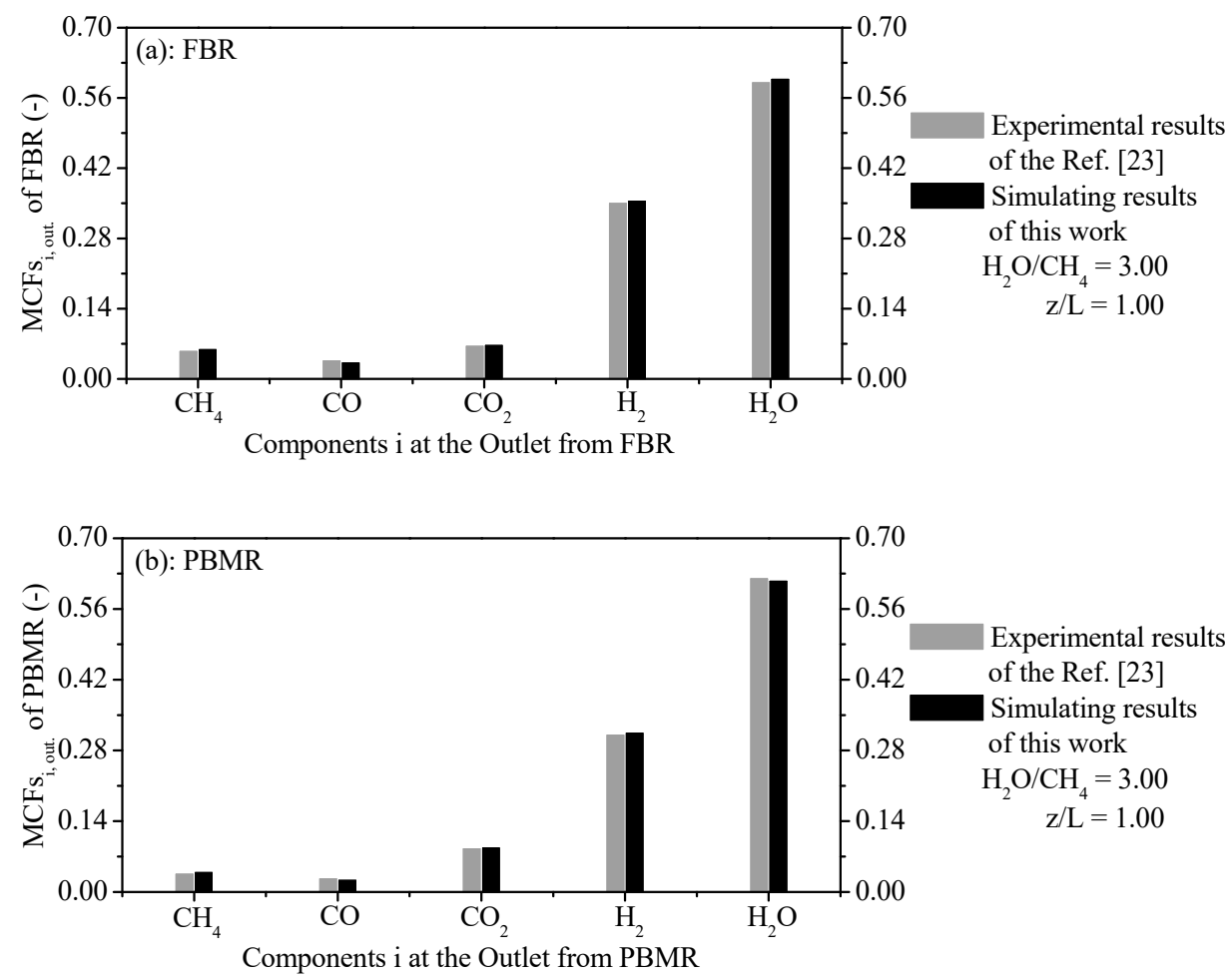

Figure 2. (a) Mole fractions of chemical components i at the outlet from Fixed Bed Reformer (FBR); (b) Mole fractions of chemical components i at the reaction zone's outlet from PBMR.

The SRs for these two cases of the MFCs $i$ are in agreement good with the literature data available in Ref. [23]. Slight differences can be found due to the deviation between the literature results and simulating results. An average relative error (ARE), Equation (57), was used to compute the consistency criterion between the results obtained and this ARE is given as follows.

$$
\text { ARE }=\left|\frac{M_{F C s_{i}^{\text {Ref.[23] }}}-\text { MFCs }_{i}^{\text {SRs. }}}{\operatorname{MFCs}_{i}^{\text {Ref. }[23]}}\right| \times 100 ; \mathrm{i}=\mathrm{CH}_{4}, \mathrm{H}_{2} \mathrm{O}, \mathrm{H}_{2}, \mathrm{CO} \text { and } \mathrm{CO}_{2}
$$

The good accordance between the SRs and literature data show that the developed model is acceptable. Considering the studied cases in Figure 2a,b, we obtained consistent satisfactory results from MFCs i against experimental results from the literature [23], resulting in AREs of 7.35\% $\leq \mathrm{ARE}_{\mathrm{CH}_{4}} \leq 11.25 \%, 7.79 \% \leq \mathrm{ARE}_{\mathrm{H}_{2} \mathrm{O}} \leq 11.68 \%, 1.85 \% \leq$ $\mathrm{ARE}_{\mathrm{H}_{2}} \leq 4.07 \%, 0.65 \% \leq \mathrm{ARE}_{\mathrm{CO}} \leq 3.23 \%$, and $1.13 \% \leq \mathrm{ARE}_{\mathrm{CO}_{2}} \leq 3.75 \%$ to $\mathrm{FBR}$ and $5.03 \% \leq \mathrm{ARE}_{\mathrm{CH}_{4}} \leq 8.05 \%, 6.71 \leq \mathrm{ARE}_{\mathrm{H}_{2} \mathrm{O}} \leq 9.73 \%, 1.12 \leq \mathrm{ARE}_{\mathrm{H}_{2}} \leq 4.11 \%$, $0.36 \% \leq \mathrm{ARE}_{\mathrm{CO}} \leq 2.64 \%$ and $0.31 \% \leq \mathrm{ARE}_{\mathrm{CO}_{2}} \leq 2.69 \%$ for PBMR.

The CIEA method has been used to simulate the results of the SRM method in PBMR and FBR. The CIEA method can be considered as a potential candidate for solving an NPDE system at lower CPU time. Table 5 shows the results of the SRM method in PBMR and FBR and, thus, these results are compared with the Finite Volume (FV) method against the CIEA method. 
Table 5. Comparison of the results between the Finite Volume (FV) method and Coupled Integral Equation Approach (CIEA) method.

\begin{tabular}{|c|c|c|c|c|c|c|c|c|}
\hline \multirow[b]{3}{*}{ Compts. } & \multicolumn{4}{|c|}{ FV Method } & \multicolumn{4}{|c|}{ CIEA Method } \\
\hline & \multicolumn{2}{|c|}{$\begin{array}{c}\text { PBMR } \\
\left(\mathrm{t}_{\mathrm{CPU}}=3.2 \mathrm{~h}\right)\end{array}$} & \multicolumn{2}{|c|}{$\begin{array}{c}\text { FBR } \\
\left(t_{\mathrm{CPU}}=3.4 \mathrm{~h}\right)\end{array}$} & \multicolumn{2}{|c|}{$\begin{array}{c}\text { PBMR } \\
\left(t_{\mathrm{CPU}}=2.5 \mathrm{~h}\right)\end{array}$} & \multicolumn{2}{|c|}{$\begin{array}{c}\text { FBR } \\
\left(t_{\mathrm{CPU}}=2.6 \mathrm{~h}\right)\end{array}$} \\
\hline & $\begin{array}{l}\text { Ref. } \\
\text { [23] }\end{array}$ & Teor. & $\begin{array}{l}\text { Ref. } \\
\text { [23] }\end{array}$ & Teor. & $\begin{array}{l}\text { Ref. } \\
\text { [23] }\end{array}$ & Teor. & $\begin{array}{l}\text { Ref. } \\
\text { [23] }\end{array}$ & Teor. \\
\hline $\mathrm{CH}_{4}$ & 0.035 & 0.0382 & 0.055 & 0.0586 & 0.035 & 0.0386 & 0.055 & 0.0567 \\
\hline $\mathrm{CO}$ & 0.026 & 0.0241 & 0.036 & 0.0323 & 0.026 & 0.0231 & 0.036 & 0.0319 \\
\hline $\mathrm{CO}_{2}$ & 0.085 & 0.0839 & 0.065 & 0.0667 & 0.085 & 0.0867 & 0.065 & 0.0670 \\
\hline $\mathrm{H}_{2}$ & 0.301 & 0.3101 & 0.352 & 0.3548 & 0.301 & 0.3142 & 0.352 & 0.3567 \\
\hline $\mathrm{H}_{2}$ & 0.621 & 0.6017 & 0.591 & 0.5971 & 0.621 & 0.6151 & 0.591 & 0.6001 \\
\hline
\end{tabular}

\subsection{Simulating Process}

\subsubsection{Temperature Profiles of Endothermic Reactions}

The Temperature Profiles of Endothermic Reactions (TPERs) have been computed inside FBR and within the reaction zone from PBMR and can be seen in Figure 3. It was shown that TPERs tend to assume inflection points of minimum values at which the SRM method's minimum temperatures are found due to the effect of endothermic reactions. The location of the minimum values of these TPERs could be due to the interaction of many factors as the catalytic bed's composition of the reaction zone from PBMR, initial temperature, operating pressure, and the thermodynamic equilibrium of endothermic reactions. It is clearly shown that the ERT of the FBR is much higher than the ERT in the reaction zone from PBMR. As it was reported in Figure 3, the TPER in PBMR's reaction zone reached the stable state (at about $\mathrm{z} / \mathrm{L}= \pm 0.5$ ) faster than FBR. After achieving the stable state, ERT is kept constant up to $\mathrm{z} / \mathrm{L}=1.0$ for FMBR. On the other hand, the TPER in FBR achieved the stable state at about $\mathrm{z} / \mathrm{L}= \pm 0.85$. As an advantage from PBMR compared to FBR, the thermodynamic equilibrium of reforming reactions in PBMR is obtained for a lesser reaction temperature due to the removal of $\mathrm{H}_{2}$ through Pd-based dense membrane.

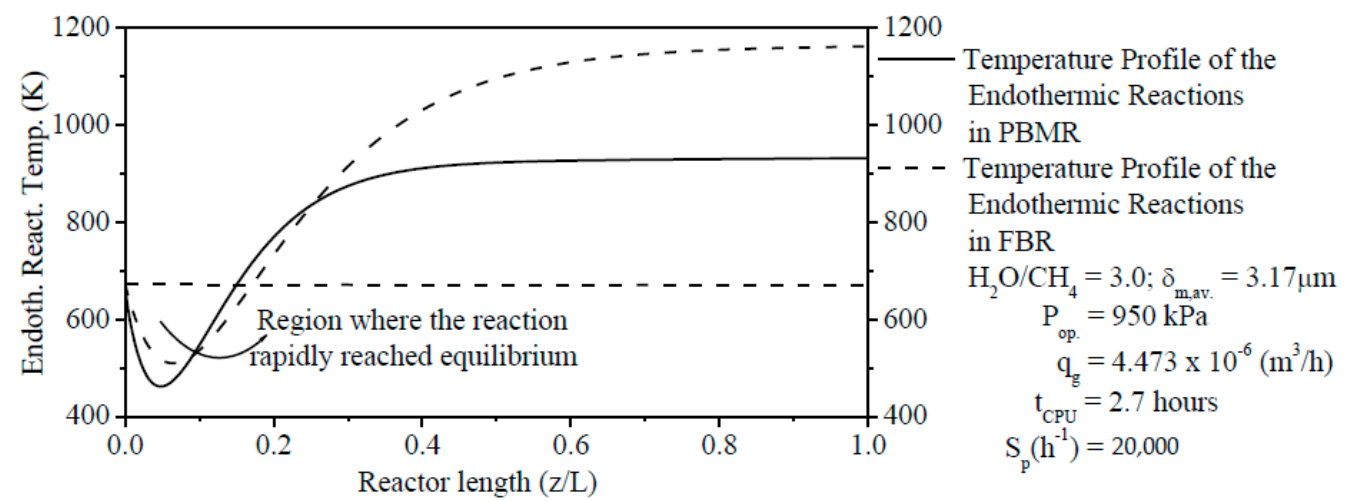

Figure 3. Comparison of the Temperature Profiles of Endothermic Reactions (TPERs) along the length of FBR and along the reaction zone length from $\mathrm{PBMR}$ on $\mathrm{Ni} / \gamma-\mathrm{Al}_{2} \mathrm{O}_{3}$.

\subsubsection{Effect of $\mathrm{H}_{2} \mathrm{O} / \mathrm{CH}_{4}$ Ratio on the ERT}

Figure 4 describes the PBMR's inlet $\mathrm{H}_{2} \mathrm{O} / \mathrm{CH}_{4}$ ratio as an important parameter on the ERT inside reaction zone from PBMR. For PBMR, the decrease of the $\mathrm{H}_{2} \mathrm{O} / \mathrm{CH}_{4}$ ratio has a negative effect on the ERT within the reaction zone. Incidentally, the main objective of PBMR is to carry out the thermochemical conversion of $\mathrm{CH}_{4}$ at moderate temperature because of the shift of the thermodynamic equilibrium on account of the removal of $\mathrm{H}_{2}$ through the membrane. Different values of the $\mathrm{H}_{2} \mathrm{O} / \mathrm{CH}_{4}$ ratio were chosen for the comparison. When the $\mathrm{H}_{2} \mathrm{O} / \mathrm{CH}_{4}$ ratio at the inlet from $\mathrm{PBMR}$ is low $\left(\mathrm{H}_{2} \mathrm{O} / \mathrm{CH}_{4}=0.95\right)$, 
the heat absorption in reaction zone section is notably increased and therefore, the ERT is favored.

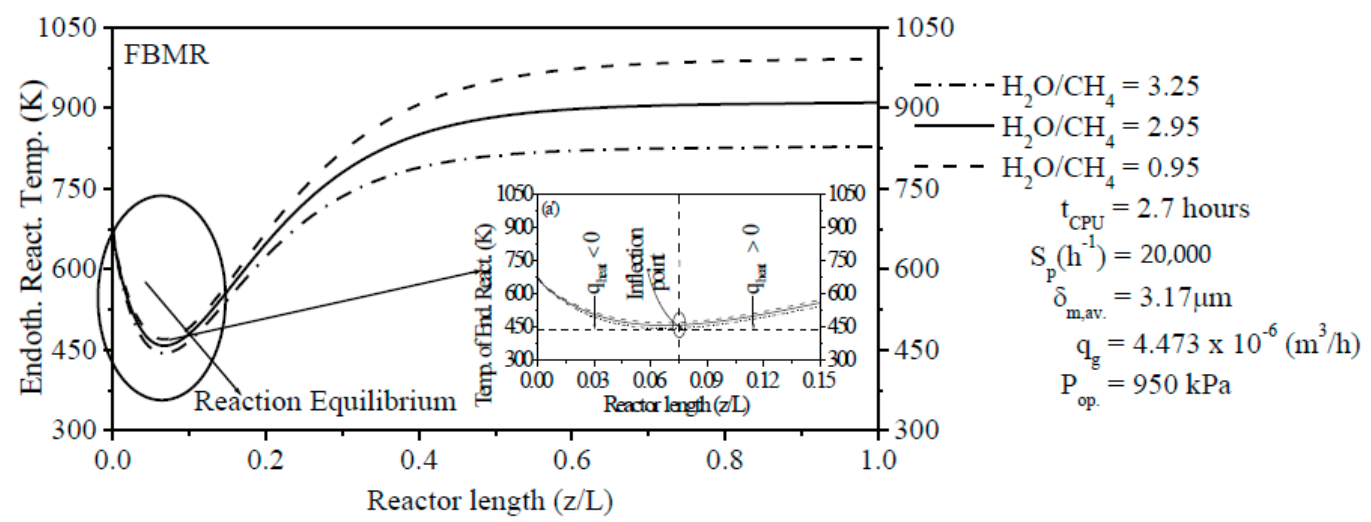

Figure 4. Effect of the $\mathrm{H}_{2} \mathrm{O} / \mathrm{CH}_{4}$ ratio on the ERT along the reaction zone length from PBMR on $\mathrm{Ni} / \gamma-\mathrm{Al}_{2} \mathrm{O}_{3}$.

On the other hand, when the $\mathrm{H}_{2} \mathrm{O} / \mathrm{CH}_{4}$ ratio at the inlet is high $\left(\mathrm{H}_{2} \mathrm{O} / \mathrm{CH}_{4}=3.25\right)$, the heat absorption in the reaction zone is remarkably decreased and, thus, the ERT is reduced. It is clearly shown that the ERT attains the stable state at about $\mathrm{z} / \mathrm{L}= \pm 0.70$ and only a part of the reformer is utilized. After reaching the stable state, ERTs are maintained constant up

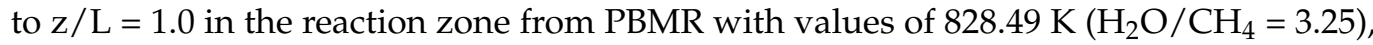
$910.27 \mathrm{~K}\left(\mathrm{H}_{2} \mathrm{O} / \mathrm{CH}_{4}=2.95\right)$, and $991.68 \mathrm{~K}\left(\mathrm{H}_{2} \mathrm{O} / \mathrm{CH}_{4}=0.95\right)$, respectively. Figure 4 shows a symmetry region in relation to inflection points of TPERs. Before achieving the inflection points, there is a decrease of the heat rate $\left(q_{\text {heat }}<0\right)$ due to the heat consumption for reforming reactions. After passing the inflection points, there is an increase of the heat rate $\left(q_{\text {heat }}>0\right)$ to keep the thermodynamic equilibrium of reforming reactions.

\subsubsection{Effect of the $S_{\text {sp }}$ on the ERT}

After investigating the $\mathrm{H}_{2} \mathrm{O} / \mathrm{CH}_{4}$ ratio's effect on the ERT, authors also studied the effect of the $S_{\mathrm{sp}}$ on the ERT inside reaction zone from PBMR. The $S_{\mathrm{sp}}$ is inversely proportional to the residence time. Thus, an increase of the $S_{\mathrm{sp}}$ indicates a decrease in the residence time of reactants within reaction zone from PBMR and, therefore, a reduction in the thermochemical conversion of $\mathrm{CH}_{4}$. In contrast, a decrease of the $\mathrm{S}_{\mathrm{sp}}$ indicates an increase of the residence time (higher contact time between catalyst and reactants) of reactants in reaction zone from PBMR and, thus, a rise in the thermochemical conversion of $\mathrm{CH}_{4}$. As a result, Figure 5 shows that the reduction of the $\mathrm{S}_{\mathrm{sp}}$ increases the ERT. An increase of the ERT has a positive effect on the thermochemical conversion of $\mathrm{CH}_{4}$ and yield of $\mathrm{H}_{2}$, i.e., the conversion of $\mathrm{CH}_{4}$ and yield of $\mathrm{H}_{2}$ increase with the rise of the ERT [3]. As it was analyzed in in Figure 4, three different values of the $S_{\text {sp }}$ were used to check the sensibility of the ERT to the $S_{\mathrm{sp}}$. Figure 5 reports that the $S_{\mathrm{sp}}$ is inversely proportional to the ERT, i.e., when the $S_{\mathrm{sp}}$ is lower $\left(S_{\mathrm{sp} \text {,ref. }}=15,000 \mathrm{~h}^{-1}\right)$, the ERT is notably increased. In contrast, when $S_{\mathrm{sp}}$ is higher $\left(\mathrm{S}_{\mathrm{sp}}=1.6 \mathrm{~S}_{\mathrm{sp}, \mathrm{ref}}=24,000 \mathrm{~h}^{-1}\right)$, the ERT is remarkably reduced. After achieving the stable state $(\mathrm{z} / \mathrm{L}= \pm 0.70)$, ERTs are kept constant in reaction zone up to $\mathrm{z} / \mathrm{L}=1.0$ from PBMR with values of $729.35 \mathrm{~K}\left(\mathrm{~S}_{\mathrm{sp}}=24,000 \mathrm{~h}^{-1}\right), 816.47 \mathrm{~K}\left(\mathrm{~S}_{\mathrm{sp}}=19,500 \mathrm{~h}^{-1}\right)$, and $885.98 \mathrm{~K}$ $\left(S_{\text {sp,ref. }}=15,000 \mathrm{~h}^{-1}\right)$, respectively. 


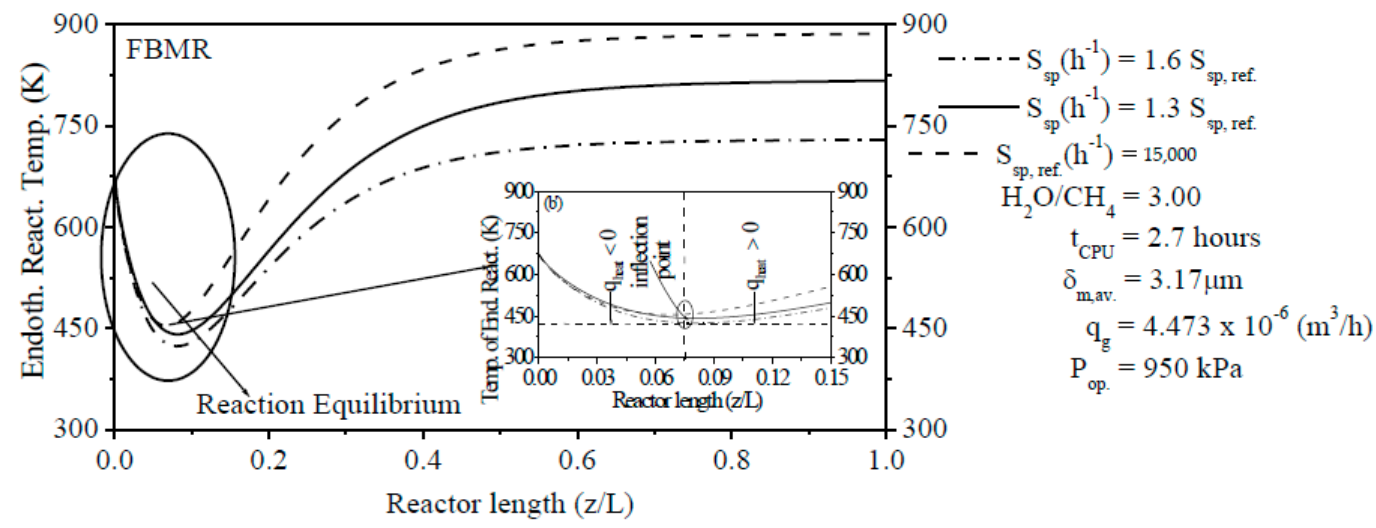

Figure 5. Effect of the $\mathrm{S}_{\mathrm{sp}}$ on the ERT along the reaction zone length from PBMR on $\mathrm{Ni} / \gamma-\mathrm{Al}_{2} \mathrm{O}_{3}$.

\subsubsection{Distribution of Chemical Components i in PBMR}

Figure 6 investigates the profiles of mole fractions for each component $i$ of the SRM method in reaction zone and permeation zone from PBMR at the following operating conditions: $\mathrm{H}_{2} \mathrm{O} / \mathrm{CH}_{4}=3.00,950 \mathrm{kPa}, 973 \mathrm{~K}$, and $4.473 \times 10^{-6}\left(\mathrm{~m}^{3} / \mathrm{h}\right)$. In this figure, the profiles of mole fractions from consumed reactants $\left(\mathrm{H}_{2} \mathrm{O}\right.$ and $\left.\mathrm{CH}_{4}\right)$ and produced products $(\mathrm{CO}$, $\mathrm{CO}_{2}$ and $\mathrm{H}_{2}$ ) have been reported in the reaction zone as well as the profile of mole fraction of $\mathrm{H}_{2}$ in the permeation zone. $\mathrm{H}_{2} \mathrm{O}$ was not fully consumed for the SRM method within the reaction zone from PBMR, i.e., after reaching a stable consumption $(\mathrm{z} / \mathrm{L}= \pm 0.17)$, only $18.67 \%$ was spent. On the other hand, $\mathrm{CH}_{4}$ was completely consumed for the SRM method inside the reaction zone from PBMR, after achieving a stable consumption $(\mathrm{z} / \mathrm{L}= \pm 0.37)$, an amount of $97.26 \%$ was consumed. After reaching the stable mole fractions $(\mathrm{z} / \mathrm{L}= \pm 0.52)$ of $\mathrm{H}_{2}$ in reaction zone and permeation zone, we obtained quantities of $29.49 \%$ (reaction zone) and $29.48 \%$ (permeation zone), respectively. Similarly, after attaining the stable mole fractions of $\mathrm{CO}_{2}(\mathrm{z} / \mathrm{L}= \pm 0.57)$ and $\mathrm{CO}(\mathrm{z} / \mathrm{L}= \pm 0.4)$ in the reaction zone, we computed the amounts of $14.23 \%$ and $2.76 \%$, respectively.

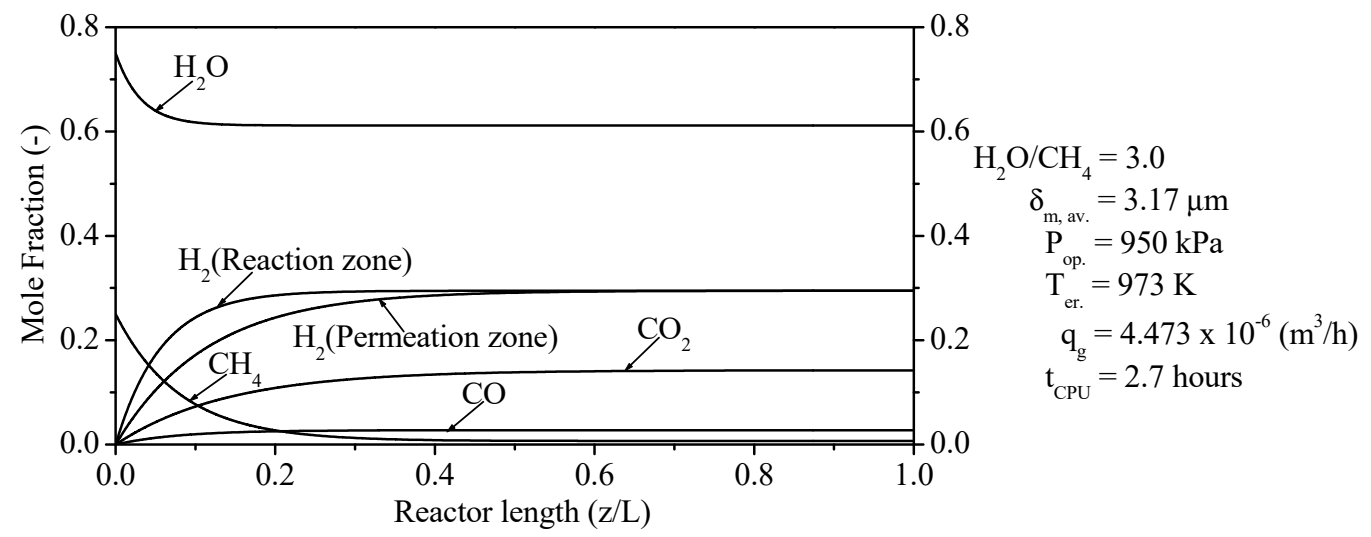

Figure 6. Molar fractions of components $\mathrm{i}$ along the reaction zone length from $\mathrm{PBMR}$ on $\mathrm{Ni} / \gamma-\mathrm{Al}_{2} \mathrm{O}_{3}$.

\subsubsection{Conversion Rate of $\mathrm{CH}_{4}$ and Feed-Based Yield of $\mathrm{H}_{2}$ in FBR and FDMR}

Thermodynamic limitations of FBRs are considered as a great problem to increase the thermochemical conversion of the SRM method. To solve this gap, we used PBMRs as innovating equipment that act with permselective membranes to overcome thermodynamic limitations and thus get a high conversion rate of $\mathrm{CH}_{4}$ at lower temperature [24]. The thermochemical performance of FBR and PBMR is analyzed from the Conversion Rate (CR) of $\mathrm{CH}_{4}$, and Feed-Based Yield (FBY) of $\mathrm{H}_{2}$ as follows.

$$
\text { Conversion Rate }(\mathrm{CR}) \text { of } \mathrm{CH}_{4}=1-\frac{\mathrm{F}_{\mathrm{CH}_{4} \text {,out }}}{\mathrm{F}_{\mathrm{CH}_{4}, 0}}
$$




$$
\text { Feed - based yield (FBY) of } \mathrm{H}_{2}=\frac{\mathrm{F}_{\mathrm{H}_{2} \text {,out. }}-\mathrm{F}_{\mathrm{H}_{2} \text {,in. }}}{\mathrm{F}_{\mathrm{H}_{2} \mathrm{O} \text {,in. }}+2 \mathrm{~F}_{\mathrm{CH}_{4} \text {, in. }}}
$$

Figure 7a compares the $\mathrm{CR}$ of $\mathrm{CH}_{4}$ inside $\mathrm{FBR}$ and $\mathrm{CR}$ of $\mathrm{CH}_{4}$ in reaction zone from PBMR. It is clearly shown that the thermochemical CR rate of $\mathrm{CH}_{4}$ in $\mathrm{FBR}$ is lower while a substantial improvement in the thermochemical CR of $\mathrm{CH}_{4}$ is achieved by PBMR. As a result, after achieving the stable state at about $\mathrm{z} / \mathrm{L}= \pm 0.20$ (see Figure $7 \mathrm{a}$ ), the $\mathrm{CR}$ of $\mathrm{CH}_{4}$ is kept constant until $\mathrm{z} / \mathrm{L}=1.0$ of FBR with a value of \pm 0.58 at the operating conditions of $\mathrm{H}_{2} \mathrm{O} / \mathrm{CH}_{4}=3.00,950 \mathrm{kPa}, 973 \mathrm{~K}$, and $4.473 \times 10^{-6} \mathrm{~m}^{3} / \mathrm{h}$. A higher $\mathrm{CR}$ of $\mathrm{CH}_{4}$ in reaction zone from PBMR is favored by the shift in the thermodynamic equilibrium according to LeChatelier's principle, sweep gas flux, change of concentration, operating temperature, and reduction of the partial pressure of $\mathrm{H}_{2}$ in the separation side. On the other hand, it is clearly shown that the $\mathrm{CR}$ of $\mathrm{CH}_{4}$ in reaction zone from PBMR is remarkably enhanced. After reaching the stable state at about $\mathrm{z} / \mathrm{L}= \pm 0.80$ (see Figure $7 \mathrm{a}$ ), the $\mathrm{CR}$ of $\mathrm{CH}_{4}$ is maintained constant up to $\mathrm{z} / \mathrm{L}=1.0$ in reaction zone from PBMR with a value of \pm 0.95 at the same operating conditions of FBR.
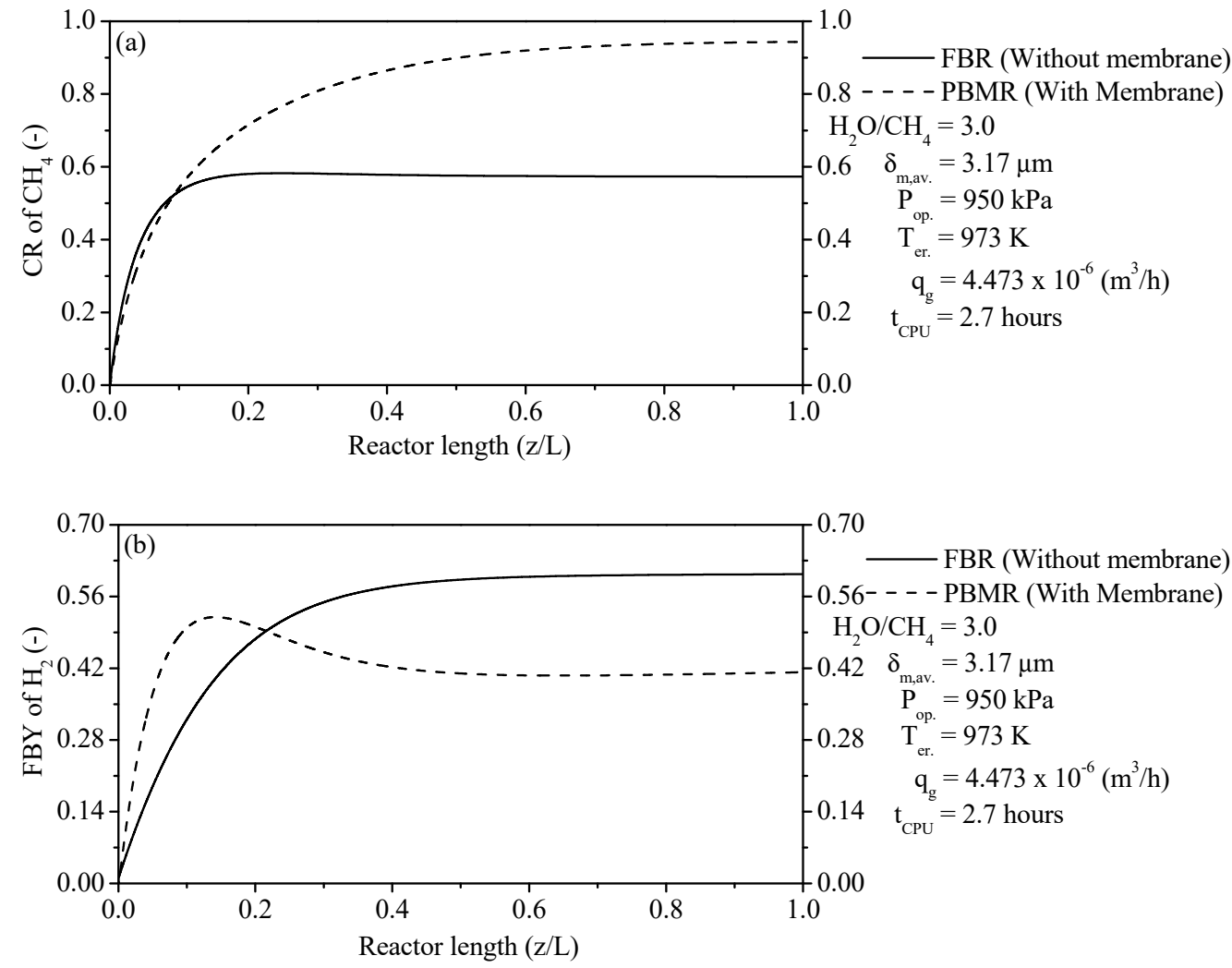

Figure 7. (a) Conversion Rate (CR) of $\mathrm{CH}_{4}$ for $\mathrm{FBR}$ and $\mathrm{CR}$ of $\mathrm{CH}_{4}$ in reaction zone from PBMR; (b) Feed-Based Yield (FBY) of $\mathrm{H}_{2}$ for FBR and FBY of $\mathrm{H}_{2}$ in reaction zone from PBMR.

Figure $7 \mathrm{~b}$ confronts the FBY of $\mathrm{H}_{2}$ within FBR and $\mathrm{FBY}$ of $\mathrm{H}_{2}$ in reaction zone from PBMR. It can be seen that the profiles of the FBY of $\mathrm{H}_{2}$ in two studied reformers have different behavior. As a result, the FBY of $\mathrm{H}_{2}$ for FBR rose notably until reaching a stable state. After that, it was kept constant up to $\mathrm{z} / \mathrm{L}=1.0$ with a value of \pm 0.61 at the same operating conditions from Figure 7a. On the other hand, the FBY of $\mathrm{H}_{2}$ for PBMR tends to take on an inflection point of a maximum value at which the effective $\mathrm{FBY}$ of $\mathrm{H}_{2}$ is optimum. After attaining the maximum value at $\mathrm{z} / \mathrm{L}= \pm 0.1$, the $\mathrm{FBY}$ of $\mathrm{H}_{2}$ decreases up to $\mathrm{z} / \mathrm{L}= \pm 0.50$ and after that, it is maintained until $\mathrm{z} / \mathrm{L}=1.0$ with a value of \pm 0.41 at the same operating conditions from Figure 7a. The profile from the FBY of $\mathrm{H}_{2}$ reports this behavior due to the removal of $\mathrm{H}_{2}$ through the membrane into the permeation zone from PBMR. 


\subsubsection{Effect of the $\mathrm{S}_{\mathrm{sp}}$ on the $\mathrm{CR}$ of $\mathrm{CH}_{4}$}

The process of reforming reactions of $\mathrm{CH}_{4}$ includes the adsorption of the reactant composition in the gaseous phase, desorption of mixture gas, and residence time of gaseous reactants.

As the $S_{\mathrm{sp}}$ is inversely proportional to the residence time, an increase on the $S_{\mathrm{sp}}$ will reduce the residence time for the gaseous reactants on the catalyst particles. Conversely, a reduction of the $S_{\text {sp }}$ points to an increase of the residence time of gaseous reactants to react on the catalyst particles and, thus, a rise in the $\mathrm{CR}_{\text {of }} \mathrm{CH}_{4}$. In order to understand the effect of the $S_{s p}$ on the CR of $\mathrm{CH}_{4}$ (see Equation (58)), simulations are carried out for different $S_{\text {sp }}$ values $\left(\mathrm{S}_{\text {sp,ref. }}=15,000 \mathrm{~h}^{-1}, \mathrm{~S}_{\mathrm{sp}}=1.3 \mathrm{~S}_{\text {sp,ref. }}=19,500 \mathrm{~h}^{-1}\right.$, and $\left.\mathrm{S}_{\mathrm{sp}}=1.6 \mathrm{~S}_{\mathrm{sp}, \text { ref. }}=24,000 \mathrm{~h}^{-1}\right)$. As it can be seen in Figure 8, the CR of $\mathrm{CH}_{4}$ is reduced to be at $\mathrm{z} / \mathrm{L}=1.0$ with the values of $16.32 \%\left(\mathrm{~S}_{\mathrm{sp}}=24,000 \mathrm{~h}^{-1}\right), 48.28 \%\left(\mathrm{~S}_{\mathrm{sp}}=19,500 \mathrm{~h}^{-1}\right)$, and $93.21 \%\left(\mathrm{~S}_{\mathrm{sp}, \text { ref. }}=15,000 \mathrm{~h}^{-1}\right)$, respectively.

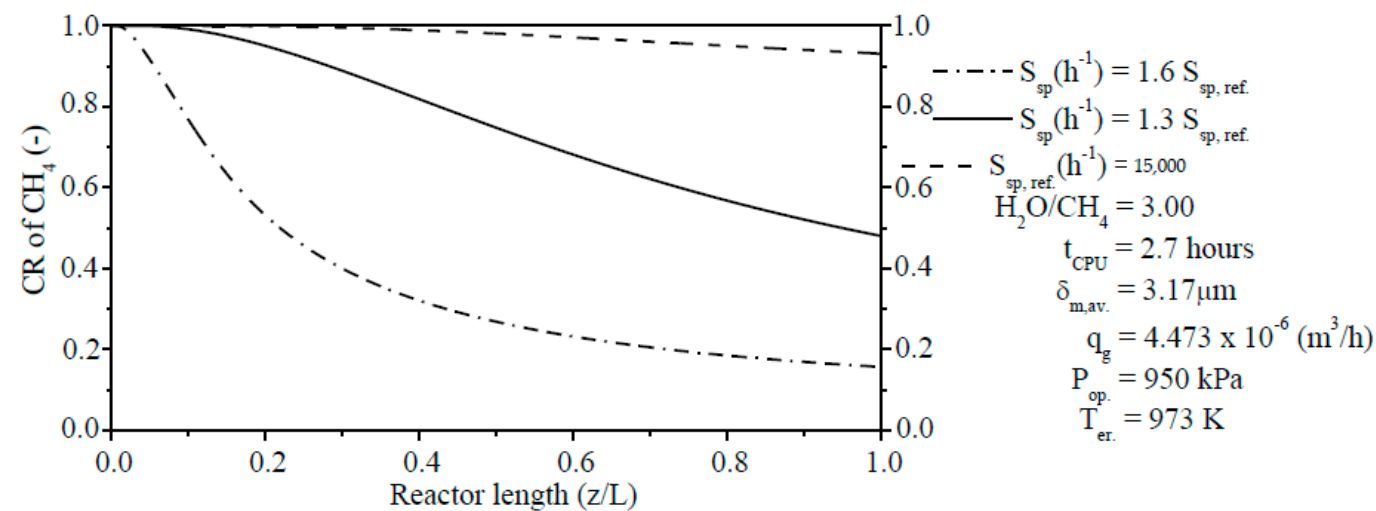

Figure 8. Effect of the $\mathrm{S}_{\mathrm{sp}}$ on the $\mathrm{CR}$ of $\mathrm{CH}_{4}$ along the reaction zone length from $\mathrm{PBMR}$ on $\mathrm{Ni} / \gamma$ $\mathrm{Al}_{2} \mathrm{O}_{3}$.

3.3.7. Effect of $\mathrm{H}_{2} \mathrm{O} / \mathrm{CH}_{4}$ Ratio on the $\mathrm{CR}$ of $\mathrm{CH}_{4}$ and Consumption-Based Yield of $\mathrm{H}_{2}$

The effect of the $\mathrm{H}_{2} \mathrm{O} / \mathrm{CH}_{4}$ ratio plays a very significant function in the $\mathrm{CR}$ of $\mathrm{CH}_{4}$ and $\mathrm{CBY}$ of $\mathrm{H}_{2}$ in the reaction zone from PBMR. The results of the $\mathrm{CR}$ of $\mathrm{CH}_{4}$ in Figure $9 \mathrm{a}$ have been computed using Equation (58) from the above item. On the other hand, results of the $\mathrm{CBY}$ of $\mathrm{H}_{2}$ in Figure 9b were obtained through Equation (60) as follows.

$$
\text { Consumption - based yield }(\mathrm{CBY}) \text { of } \mathrm{H}_{2}=\frac{\mathrm{F}_{\mathrm{H}_{2}, \text { out. }}-\mathrm{F}_{\mathrm{H}_{2} \text {,in. }}}{\left(\mathrm{F}_{\mathrm{H}_{2} \mathrm{O} \text {,in. }}+2 \mathrm{~F}_{\mathrm{CH}_{4} \text {, in. }}\right)-\left(\mathrm{F}_{\mathrm{H}_{2} \mathrm{O} \text {,out. }}+2 \mathrm{~F}_{\mathrm{CH}_{4} \text {, out. }}\right)}
$$

Figure 9a shows the profiles of the $\mathrm{CR}$ of $\mathrm{CH}_{4}$ at three different values of the $\mathrm{H}_{2} \mathrm{O} / \mathrm{CH}_{4}$ ratio in reaction zone from PBMR. The $\mathrm{CR}$ of $\mathrm{CH}_{4}$ can be notably reduced with the increase of the coke formation on Ni catalysts. Based on the profiles from Figure 9a, it has been noted that an increase of the $\mathrm{H}_{2} \mathrm{O} / \mathrm{CH}_{4}$ ratio raises the $\mathrm{CR}$ of $\mathrm{CH}_{4}$ due to the reduction of the coke content on the external surface of the catalyst particles. On the other hand, a decrease of the $\mathrm{H}_{2} \mathrm{O} / \mathrm{CH}_{4}$ ratio reduces the $\mathrm{CR}$ of $\mathrm{CH}_{4}$ owing to the increase of the coke formation on the outside surface of catalyst particles. As a result, the $\mathrm{CR}$ of $\mathrm{CH}_{4}$ was reduced to be at $\mathrm{z} / \mathrm{L}=1.0$ with the values of $94.75 \%\left(\mathrm{H}_{2} \mathrm{O} / \mathrm{CH}_{4}=3.25\right), 86.57 \%\left(\mathrm{H}_{2} \mathrm{O} / \mathrm{CH}_{4}\right.$ $=2.95)$, and $72.06 \%\left(\mathrm{H}_{2} \mathrm{O} / \mathrm{CH}_{4}=0.95\right)$, respectively.

Figure $9 \mathrm{~b}$ reports the profiles of the $\mathrm{CBY}$ of $\mathrm{H}_{2}$ (Equation (60)) at three different values of the $\mathrm{H}_{2} \mathrm{O} / \mathrm{CH}_{4}$ ratio in reaction zone from PBMR. Unlike of the $\mathrm{CR}$ of $\mathrm{CH}_{4}$, the profiles of the $\mathrm{CBY}$ of $\mathrm{H}_{2}$ sharply decrease with the increase of the $\mathrm{H}_{2} \mathrm{O} / \mathrm{CH}_{4}$ ratio. An increase of steam content has a negative effect on the production of $\mathrm{H}_{2}$, i.e., the generating rate of $\mathrm{H}_{2}$ isn't strengthened according to Figure 9b. The profiles of the CBY of $\mathrm{H}_{2}$ tend to take on inflection points of maximum values at which the effective $\mathrm{CBYs}$ of $\mathrm{H}_{2}$ is optimum. After achieving the maximum values, the CBYs of $\mathrm{H}_{2}$ decrease and after that, they are kept up to 
$\mathrm{z} / \mathrm{L}=1.0$ with values of $1.1958\left(\mathrm{H}_{2} \mathrm{O} / \mathrm{CH}_{4}=0.95\right), 0.8169\left(\mathrm{H}_{2} \mathrm{O} / \mathrm{CH}_{4}=2.95\right)$, and 0.3572 $\left(\mathrm{H}_{2} \mathrm{O} / \mathrm{CH}_{4}=3.25\right)$, respectively.
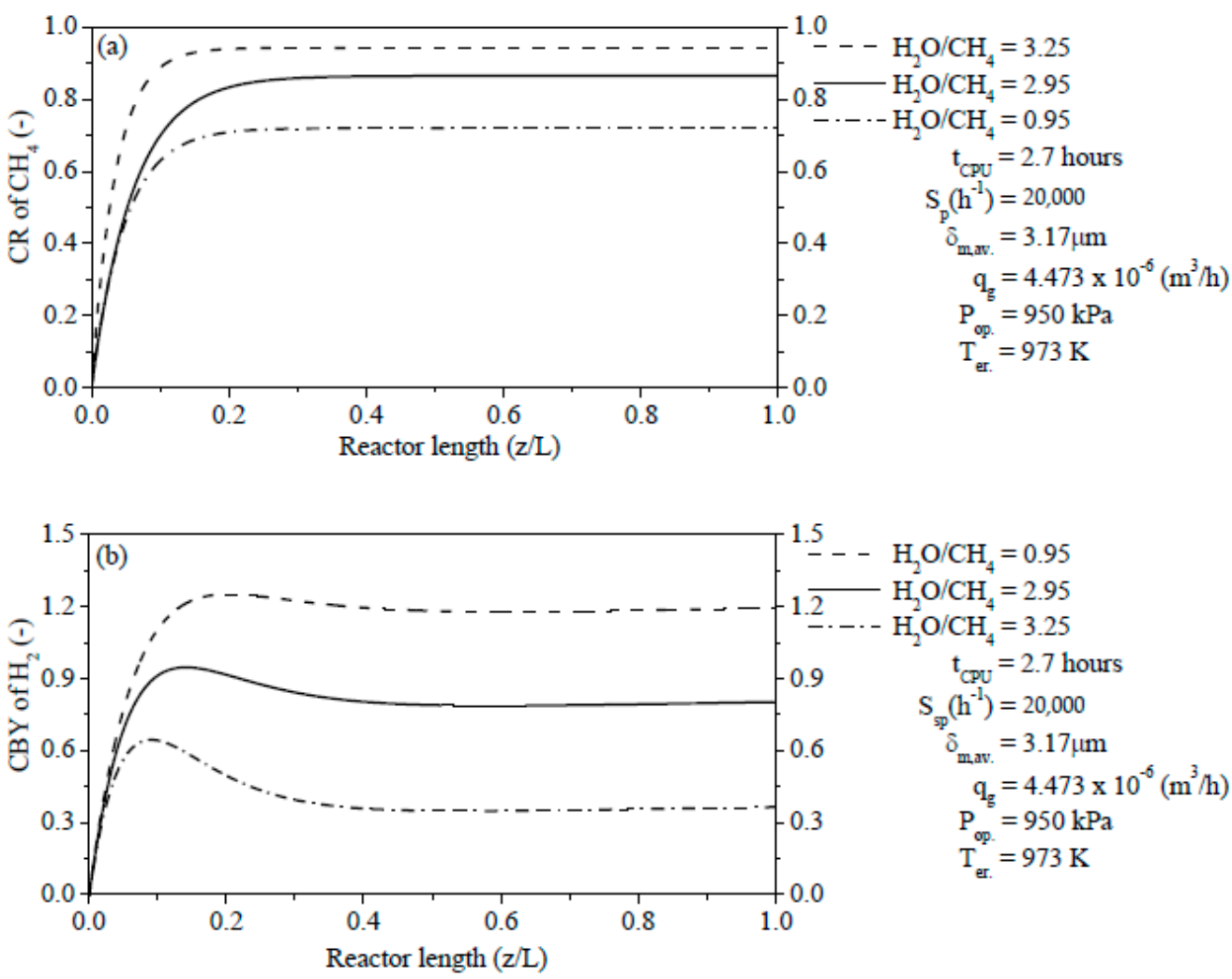

Figure 9. (a) Effect of the $\mathrm{H}_{2} \mathrm{O} / \mathrm{CH}_{4}$ ratio on the $\mathrm{CR}$ of $\mathrm{CH}_{4}$ along of the reaction zone length from PBMR; (b) Effect of the $\mathrm{H}_{2} \mathrm{O} / \mathrm{CH}_{4}$ ratio on the CBY of $\mathrm{H}_{2}$ along of the reaction zone length from PBMR.

\subsubsection{Thermochemical Energy Storage Efficiency}

The reforming reaction's thermochemical conversion of $\mathrm{CH}_{4}$ is a new technology which provides the advantage of high storage densities and minor thermal losses. The $\eta_{\text {teses }}$ in PBMRs using the SRM method play an important role in energy storage and this will be discussed in this section. The $\eta_{\text {tese }}$ of the SRM process in PBMR is computed as the ratio between the net chemical energy produced per the input electric power as follows.

$$
\eta_{\text {tese }}(\%)=\frac{Q_{\text {che. }}}{P_{\text {elet. }}}=\frac{\rho_{\mathrm{s}} \frac{\left(1-\varepsilon_{\mathrm{P}}\right)}{\varepsilon_{\mathrm{P}}} \sum_{\mathrm{j}=1}^{3}\left( \pm \Delta \mathrm{H}_{\mathrm{j}}\right) \eta_{\mathrm{j}} \mathrm{R}_{\mathrm{j}}}{\mathrm{U}_{\text {ghe }} \mathrm{S}_{\text {she }}\left(\mathrm{T}_{\text {er }}-\mathrm{T}_{\mathrm{g}}\right)}
$$

Figure 10 describes the curves of the $\eta_{\text {tese }}$ (Equation (61)) at three different values of the ERT in reaction zone from PBMR. A raise of the ERT has a positive effect on the $\eta_{\text {tese, }}$, i.e., the $\eta_{\text {tese }}$ is improved according to Figure 10. As a result, it is clearly noted that an increase of the ERT will lead to higher $\eta_{\text {teses. }}$. The curves of $\eta_{\text {teses }}$ trend to assume inflection points of maximum values $\left(P_{\text {elet. }}= \pm 150 \mathrm{~W}\right)$ in which the effective $\eta_{\text {tese }}$ are optimum. After reaching the maximum values, the $\eta_{\text {teses }}$ decrease and then they are maintained until $P_{\text {elet. }}=1000 \mathrm{~W}$ with values of $68.96 \%(E R T=1023 \mathrm{~K}), 63.21 \%(\mathrm{ERT}=973 \mathrm{~K})$, and $48.12 \%(\mathrm{ERT}=723 \mathrm{~K})$, respectively. 


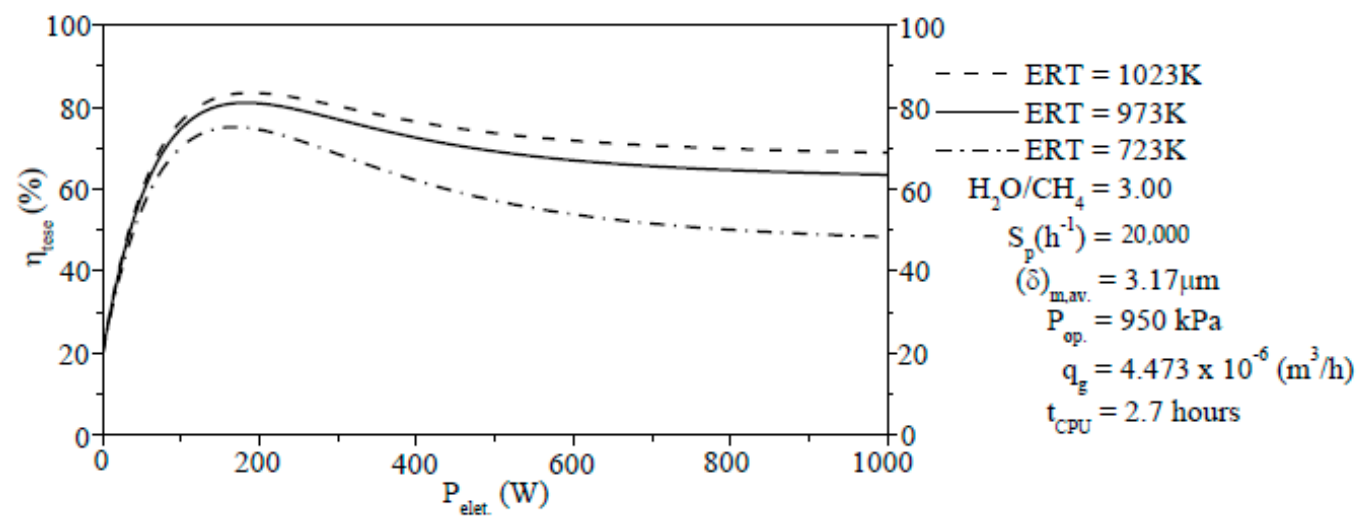

Figure 10. Effect of the ERT on the $\eta_{\text {tese }}$ of the SRM method in PBMR on Ni/Mg/ $\gamma-\mathrm{A} \ell_{2} \mathrm{O}_{3}$ with $6 \%$ Ni loading.

\subsubsection{Energy Storage Performance}

The energy storage's importance has motivated researchers of this work to study the energetic aspects of the storing technology as from thermochemical conversion. The chemical energy storage, sensible heat, and heat loss play important roles in the energy storage process. Figure 11 shows the energy storage performances of the thermochemical reforming method of $\mathrm{CH}_{4}$ for different reaction temperatures in reaction zone from PBMR. As it can be seen in this figure, as the ERT increases the chemical energy and the heat loss increase drastically. On the other hand, the sensible heat gradually increases as the ERT rises. After reaching the ERT of $973 \mathrm{~K}$, the chemical energy, sensible heat, and heat loss had values of $384.96 \mathrm{~W}, 151.68 \mathrm{~W}$, and $249.73 \mathrm{~W}$, respectively.

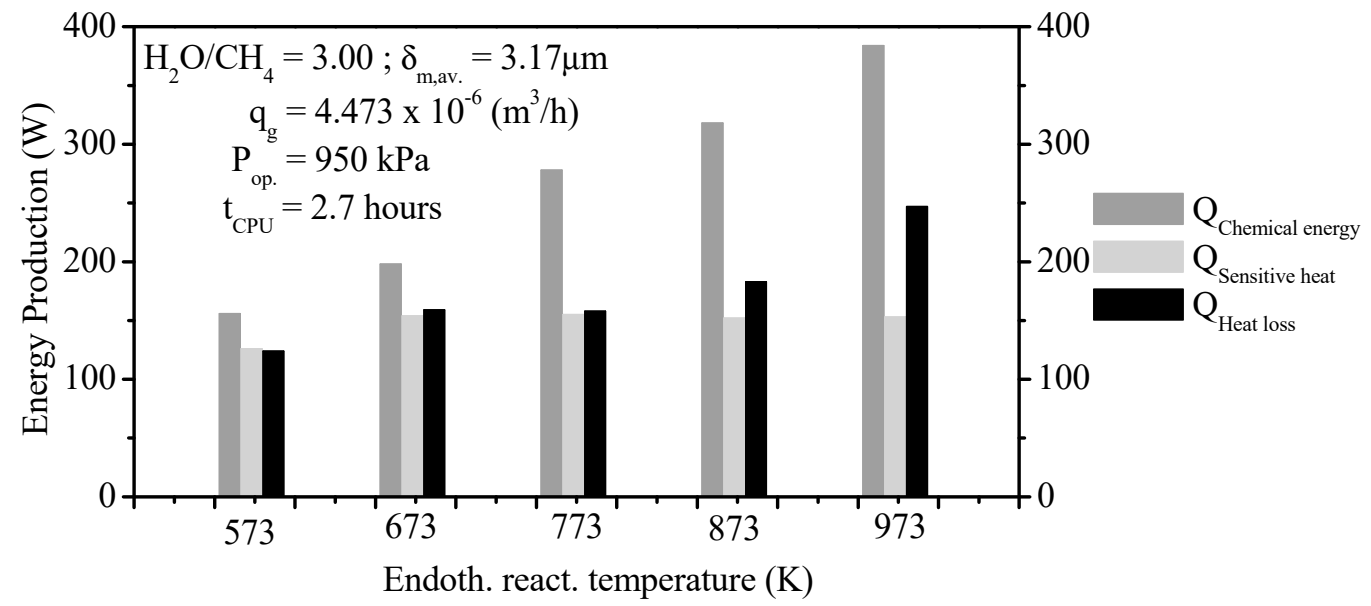

Figure 11. Energy storage performance of the thermochemical system as a function of the ERT.

\subsubsection{Selectivity of Components of the SRM Method}

The loading process of thermal energy on PBMR is used to drive the endothermic reactions of $\mathrm{CH}_{4}$. The thermal energy is thermochemically employed to convert reactants $\left(\mathrm{CH}_{4}\right.$ and $\left.\mathrm{H}_{2} \mathrm{O}\right)$ into products $\left(\mathrm{H}_{2}, \mathrm{CO}\right.$, and $\left.\mathrm{CO}_{2}\right)$. As a result, the performance of components $\left(\mathrm{CH}_{4}, \mathrm{H}_{2}, \mathrm{CO}\right.$, and $\left.\mathrm{CO}_{2}\right)$ of the SRM method were analyzed in terms of the $\mathrm{CR}$ of $\mathrm{CH}_{4}$, FBY of $\mathrm{H}_{2}, \mathrm{CBY}$ of $\mathrm{H}_{2}$, and selectivity of $\mathrm{H}_{2}, \mathrm{CO}, \mathrm{CO}_{2}$ and $\mathrm{CH}_{4}$. A set of corresponding expressions are used to compute the selectivity of $\mathrm{H}_{2}, \mathrm{CO}, \mathrm{CO}_{2}$, and $\mathrm{CH}_{4}$ as follows.

$$
\mathrm{S}_{\mathrm{H}_{2}}(\%)=\frac{\left(\mathrm{F}_{\mathrm{H}_{2}, \mathrm{rz}}^{\text {out. }}+\mathrm{F}_{\mathrm{H}_{2}, \mathrm{pz}}^{\text {out. }}\right)-\mathrm{F}_{\mathrm{H}_{2}}^{\text {in. }}}{\left(\mathrm{F}_{\mathrm{H}_{2}, \mathrm{rz}}^{\text {out. }}+\mathrm{F}_{\mathrm{H}_{2}, \mathrm{pz}}^{\text {out. }}\right)+\left(\mathrm{F}_{\mathrm{CO}, \mathrm{rz}}^{\text {out. }}+\mathrm{F}_{\mathrm{CO}_{2}, \mathrm{rz}}^{\text {out. }}+\mathrm{F}_{\mathrm{CH}_{4}, \mathrm{rz}}^{\text {out. }}\right)} \times 100
$$




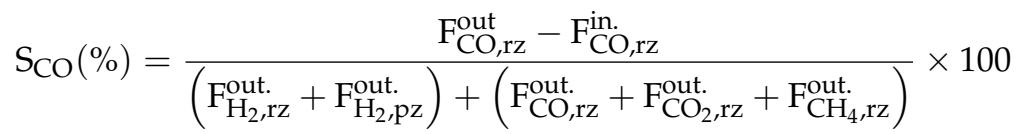

$$
\begin{aligned}
& \mathrm{S}_{\mathrm{CO}_{2}}(\%)=\frac{\mathrm{F}_{\mathrm{CO}_{2}, \mathrm{rz}}^{\text {out. }}-\mathrm{F}_{\mathrm{CO}_{2}, \mathrm{rz}}^{\text {in. }}}{\left(\mathrm{F}_{\mathrm{H}_{2}, \mathrm{rz}}^{\text {out }}+\mathrm{F}_{\mathrm{H}_{2}, \mathrm{pz}}^{\text {out. }}\right)+\left(\mathrm{F}_{\mathrm{CO}, \mathrm{rz}}^{\text {out. }}+\mathrm{F}_{\mathrm{CO}_{2}, \mathrm{rz}}^{\text {out. }}+\mathrm{F}_{\mathrm{CH}_{4}, \mathrm{rz}}^{\text {out. }}\right)} \times 100 \\
& \mathrm{~S}_{\mathrm{CH}_{4}}(\%)=\frac{\mathrm{F}_{\mathrm{CH}_{4}, \mathrm{rz}}^{\text {out }}-\mathrm{F}_{\mathrm{CH}_{4}, \mathrm{rz}}^{\text {in. }}}{\left(\mathrm{F}_{\mathrm{H}_{2}, \mathrm{rz}}^{\text {out }}+\mathrm{F}_{\mathrm{H}_{2}, \mathrm{pz}}^{\text {out. }}\right)+\left(\mathrm{F}_{\mathrm{CO}, \mathrm{rz}}^{\text {out. }}+\mathrm{F}_{\mathrm{CO}_{2}, \mathrm{rz}}^{\text {out. }}+\mathrm{F}_{\mathrm{CH}_{4}, \mathrm{rz}}^{\text {out. }}\right)} \times 100
\end{aligned}
$$

Table 6 shows the effect of the dense membrane thickness $\left(\delta_{\mathrm{m}}\right)$ on the selectivity of chemical components $\left(\mathrm{H}_{2}, \mathrm{CO}, \mathrm{CO}_{2}\right.$, and $\left.\mathrm{CH}_{4}\right)$ in PBMR. For each $\delta_{\mathrm{m}}$, we noted a different value of the selectivity for each chemical component at same operating conditions: $\mathrm{H}_{2} \mathrm{O} / \mathrm{CH}_{4}=3.00,950 \mathrm{kPa}, 973 \mathrm{~K}$, and $4.473 \times 10^{-6} \mathrm{~m}^{3} / \mathrm{h}$. As a result, $\mathrm{H}_{2}$ presented higher values of the selectivity varying from $60.98 \%$ to $73.18 \%$ while $\mathrm{CH}_{4}$ reported lower values of the selectivity ranging from $1.41 \%$ to $2.06 \%$. On the other hand, CO presented corresponding values of the selectivity from $10.71 \%$ to $15.15 \%$, whilst $\mathrm{CO}_{2}$ has related relative values of the selectivity ranging from $14.69 \%$ to $21.37 \%$.

\begin{tabular}{|c|c|c|c|c|c|}
\hline \multirow[b]{2}{*}{$\delta_{m}(\mu \mathrm{m})$} & \multicolumn{5}{|c|}{$\operatorname{PBMR}\left(\mathrm{H}_{2} \mathrm{O} / \mathrm{CH}_{4}=3.00, \mathrm{P}_{\mathrm{zr}}=950 \mathrm{kPa}, \mathrm{P}_{\mathrm{pz}}=150 \mathrm{kPa}\right.$, and ERT $\left.=973 \mathrm{~K}\right)$} \\
\hline & 1.7 & 2.7 & 3.7 & 4.7 & 5.7 \\
\hline \multicolumn{6}{|c|}{ Selectivities } \\
\hline $\mathrm{S}_{\mathrm{H}_{2}}(\%)$ & 60.98 & 73.18 & 68.39 & 64.78 & 61.95 \\
\hline $\mathrm{S}_{\mathrm{CO}}(\%)$ & 15.17 & 10.71 & 12.62 & 14.06 & 15.19 \\
\hline $\mathrm{S}_{\mathrm{CO}_{2}}(\%)$ & 21.37 & 14.69 & 17.31 & 19.29 & 20.84 \\
\hline $\mathrm{S}_{\mathrm{CH}_{4}}(\%)$ & 2.06 & 1.41 & 1.67 & 1.85 & 2.03 \\
\hline
\end{tabular}

Table 6. Selectivity of $\mathrm{H}_{2}, \mathrm{CO}, \mathrm{CO}_{2}$, and $\mathrm{CH}_{4}$ as a function of the dense membrane thicknesses $\left(\delta_{\mathrm{m}}\right)$.

\section{Conclusions and Future Work}

The present work has been focused on a numerical analysis of physical-mathematical modelling and computer simulation to describe the performance of reformers for the production of $\mathrm{H}_{2}$ using a reference method of steam reforming $\mathrm{CH}_{4}$. The model equations that describe the gas temperature in the reaction zone, endothermic reaction's temperature in the reaction zone, molar flow of the components $i$ in the reaction zone, molar flow of $\mathrm{H}_{2}$ in the reaction zone, and molar flow of $\mathrm{H}_{2}$ in the permeation zone have been reported and discussed. As a solution to the model equations, the main focus has been the CIEA method as a powerful technique to reduce the NPDE system of this work into a NODE system using the boundary conditions of each NPDE. The work's results highlighted the importance of the mathematical model developed to describe the performance from FBR and PBMR. In this context, the main conclusions are summarized as follows.

1. The computed results of MFCs are compared with experimental results from the literature under same operating conditions for FBR and PBMR. Figure 2a compares the computed results of MFCs against experimental results of MFCs of components $\mathrm{CH}_{4}, \mathrm{CO}, \mathrm{CO}_{2}, \mathrm{H}_{2}$, and $\mathrm{H}_{2} \mathrm{O}$ at the outlet from $\mathrm{FBR}$ with AREs around $7.35 \% \leq$ $\mathrm{ARE}_{\mathrm{CH}_{4}} \leq 11.25 \%, 7.79 \% \leq \mathrm{ARE}_{\mathrm{H}_{2} \mathrm{O}} \leq 11.68 \%, 1.85 \% \leq \mathrm{ARE}_{\mathrm{H}_{2}} \leq 4.07 \%, 0.65 \% \leq$ $\mathrm{ARE}_{\mathrm{CO}} \leq 3.23 \%$, and $1.13 \% \leq \mathrm{ARE}_{\mathrm{CO}_{2}} \leq 3.75 \%$, respectively. On the other hand, Figure $2 \mathrm{~b}$ also confronts the simulated results of MFCs against the experimental results of the MFCs of components $\mathrm{CH}_{4}, \mathrm{CO}, \mathrm{CO}_{2}, \mathrm{H}_{2}$, and $\mathrm{H}_{2} \mathrm{O}$ at the outlet from FMBR with AREs of about 5.03\% $\leq \mathrm{ARE}_{\mathrm{CH}_{4}} \leq 8.05 \%, 6.71 \leq \mathrm{ARE}_{\mathrm{H}_{2} \mathrm{O}} \leq 9.73 \%$, $1.12 \leq \mathrm{ARE}_{\mathrm{H}_{2}} \leq 4.11 \%, 0.36 \% \leq \mathrm{ARE}_{\mathrm{CO}} \leq 2.64 \%$ and $0.31 \% \leq \mathrm{ARE}_{\mathrm{CO}_{2}} \leq 2.69 \%$, respectively.

2. The thermochemical conversion of $\mathrm{CH}_{4}$ and production of $\mathrm{H}_{2}$ are considered the two main parameters of FBR and PBMR. The ERT has a positive effect on these two 
parameters. When comparing the two reformer types, the production of $\mathrm{H}_{2}$ and thermochemical conversion of $\mathrm{CH}_{4}$ on the PBMR can be obtained at lower ERT due to the removal of $\mathrm{H}_{2}$ through a membrane from PBMR. This point is a great advantage from PBMR in relation to FBR because PBMR can be operated at lower ERT than FBR and to obtain higher results of these two parameters.

3. The steam content has an important effect on the ERT in PBMR, i.e., when the $\mathrm{H}_{2} \mathrm{O} / \mathrm{CH}_{4}$ ratio is low, the ERT is notably increased. In contrast, when the $\mathrm{H}_{2} \mathrm{O} / \mathrm{CH}_{4}$ ratio is high, the ERT is remarkably decreased. The $S_{\mathrm{sp}}$ has also a significant effect on the ERT and CR of $\mathrm{CH}_{4}$. Similar to steam content, when the $\mathrm{S}_{\mathrm{sp}}$ is small, the ERT is remarkably increased. On the other hand, the ERT is reduced with the increase of the $\mathrm{S}_{\mathrm{sp}}$. For a lower Ssp value, we observed a higher CR of $\mathrm{CH}_{4}$ in PBMR. Conversely, a higher $\mathrm{S}_{\mathrm{sp}}$ value points to a low $\mathrm{CR}$ of $\mathrm{CH}_{4}$ in PBMR.

4. The effect of the $\mathrm{H}_{2} \mathrm{O} / \mathrm{CH}_{4}$ ratio played a significant role on the $\mathrm{CR}$ of $\mathrm{CH}_{4}$ and $\mathrm{CBY}$ of $\mathrm{H}_{2}$. An increase of the $\mathrm{H}_{2} \mathrm{O} / \mathrm{CH}_{4}$ ratio is associated with a significant improvement due to the coke reduction on the catalytic particles, i.e., there is a rise of the CR of $\mathrm{CH}_{4}$. Conversely, a decrease of the $\mathrm{H}_{2} \mathrm{O} / \mathrm{CH}_{4}$ ratio has a poor effect owing to the increase of the coke formation and thus the $\mathrm{CR}$ of $\mathrm{CH}_{4}$ is reduced. Unlike the $\mathrm{CR}$ of $\mathrm{CH} 4$, a rise of the $\mathrm{H}_{2} \mathrm{O} / \mathrm{CH}_{4}$ ratio led to a decrease of the $\mathrm{CBY}$ of $\mathrm{H}_{2}$. On the other hand, a reduction of the $\mathrm{H}_{2} \mathrm{O} / \mathrm{CH}_{4}$ ratio led to an increase of the $\mathrm{CBY}$ of $\mathrm{H}_{2}$.

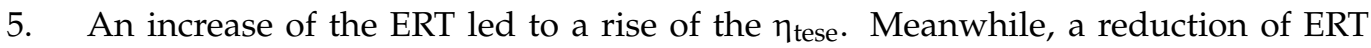
resulted in an decrease of the $\eta_{\text {tese }}$. A rise of the ERT has a positive effect on the chemical storage, i.e., the chemical energy gradually rose with the increase of ERT (156.01 W (573 K)-384.96 W (973 K)). The selectivity of components $\left(\mathrm{H}_{2}, \mathrm{CO}, \mathrm{CO}_{2}\right.$, and $\left.\mathrm{CH}_{4}\right)$ was computed for different $\delta_{\mathrm{m}}$. Among these components, $\mathrm{H}_{2}$ showed higher amounts $(60.98 \%$ to $73.18 \%)$ of the selectivity.

In future research, the exploration of novel materials like open-cell foams can be explored in the context of reforming reactions. Solid open-cell foams constitute a class of porous materials with low density and improved thermal properties. In addition, open-cell foams can be considered as potential candidates for catalyst support in the gassolid reaction field due to their high external surface area, high porosity, and low drop pressure. Thus, solid open-cell foams are future trends for reforming methods such as steam reforming of $\mathrm{CH}_{4}$, dry reforming of $\mathrm{CH}_{4}$, etc.

Author Contributions: This work has not been published elsewhere. We attest to the fact that all Authors reported on the title page have contributed significantly to the work, have read the manuscript, attest to the validity and legitimacy of the data and agree to its publication in the Journal of Membranes. All authors have read and agreed to the published version of the manuscript.

Funding: The authors of this paper would like to thank CNPq (National Council of Scientific and Technological Development) for the financial support given (Process 48354/2012).

Conflicts of Interest: Authors have no affiliation with any institution with a direct or indirect financial interest in the matter reported in the manuscript. However, the interest of authors is simply academic. 


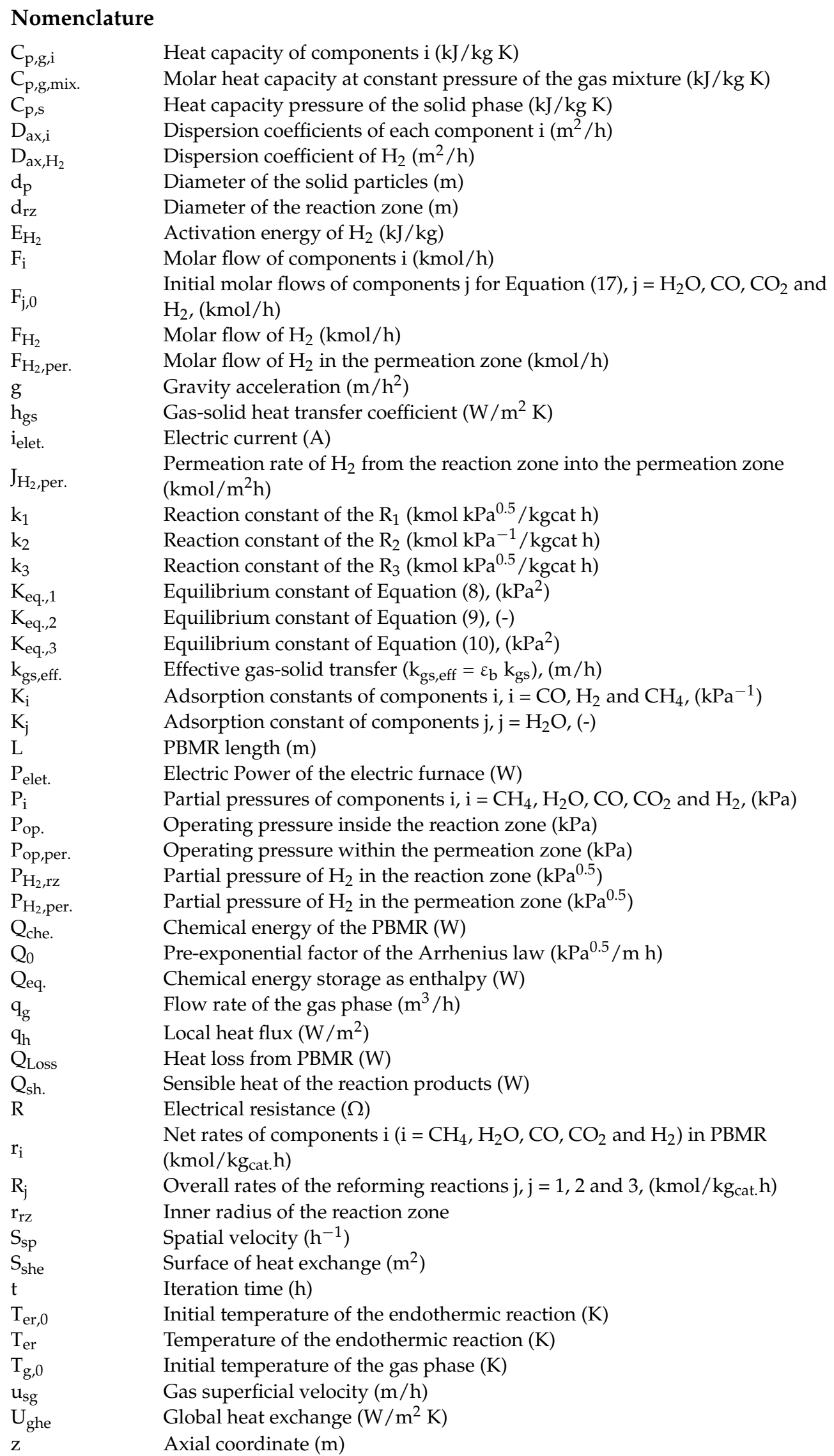




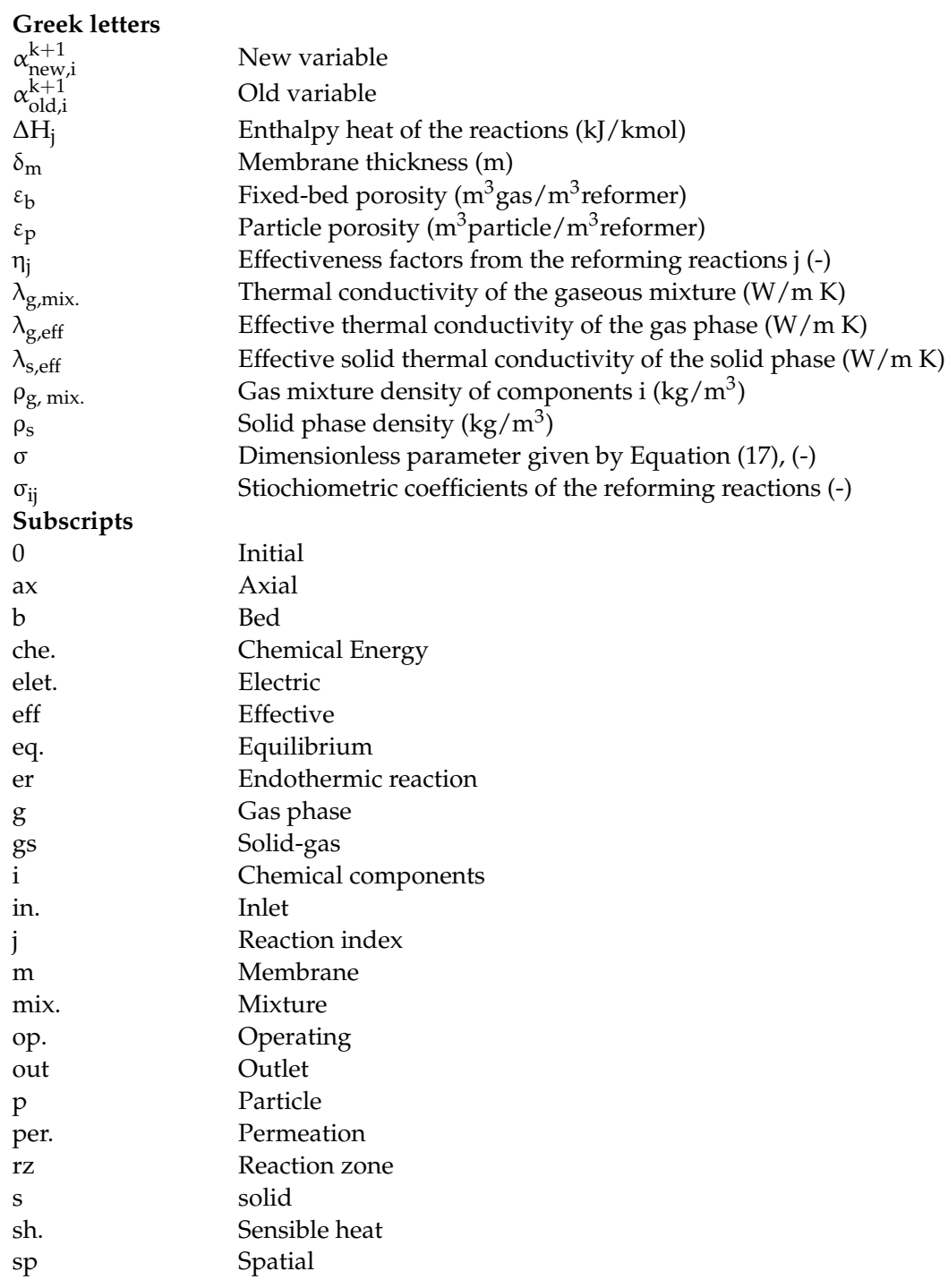

$\begin{array}{ll}\text { Abbreviations } & \\ \text { ARE } & \text { Average relative error } \\ \text { Bot. } & \text { Bottom } \\ \text { CR } & \text { Conversion rate } \\ \text { CBY } & \text { Consumption-based yield } \\ \text { CIEA } & \text { Coupled Integral Equation Approach } \\ \text { DRM } & \text { Dry reforming of methane } \\ \text { ERT } & \text { Endothermic reaction temperature } \\ \text { FBR } & \text { Fixed bed reformer } \\ \text { PBMR } & \text { Fixed bed membrane reformer } \\ \text { FBY } & \text { Food-based yield } \\ \text { K } & \text { Kelvin } \\ \text { MFC } & \text { Mole fractions of components } \\ \text { MRs } & \text { Membrane reformers } \\ \text { NIPHD } & \text { Non-isothermal pseudo-homogeneous dynamic } \\ \text { NPDE } & \text { Nonlinear Partial Differential Equation } \\ \text { NODE } & \text { Nonlinear Ordinary Differential Equation } \\ \text { SRM } & \text { Steam reforming of methane } \\ \text { SRs } & \text { Simulating results } \\ \text { TES } & \text { Thermochemical Energy Storage } \\ \text { TPER } & \text { Temperature profiles of endothermic reaction } \\ \text { Up. } & \text { Upper } \\ \text { W } & \text { Watt }\end{array}$




\section{Appendix A}

Appendix A.1. Hermite Approximation

The key idea for the coupled integral equations approximation (CIEA) has been reported using the Hermite approach [7]. The mathematical model of this work is built by a system of NPDEs for the heat transfer and mass transfer of the SRM method in PBMR. The system of NPDEs is transformed into a system of NODEs applying the CIEA method taking into account the boundary conditions of each NPDE. Expressions were used to transform PDEs into NODEs with boundary conditions, and these expressions are described as follows.

$$
\begin{gathered}
\bar{f}_{j}(t)=\frac{1}{L} \int_{0}^{L} f_{j}(z, t) d z ; j=g, s, i \\
f_{j}(L, t)-f_{j}(0, t) \cong \frac{L}{2}\left[\left.\frac{\partial f_{j}(z, t)}{\partial z}\right|_{z=0}+\left.\frac{\partial f_{j}(z, t)}{\partial z}\right|_{z=L}\right] ; j=g, s, i \\
\bar{f}_{j}(t) \cong \frac{L}{2}\left[f_{j}(0, t)+f_{j}\left(L_{z}, t\right)\right]+\frac{L^{2}}{12}\left[\left.\frac{\partial f_{j}(z, t)}{\partial z}\right|_{z=0}-\left.\frac{\partial f_{j}(z, t)}{\partial z}\right|_{z=L}\right] ; j=g, s, i
\end{gathered}
$$

Appendix A.2. CIEA Method's Application to the Energy Balance of the Gas Phase in Reaction Zone from $P B M R$

The boundary conditions (Equations (28) and (29)) of the energy balance of the gas phase at the inlet and outlet of the reaction zone from PBMR have been used to transform Equation (24) into Equation (47). The coefficients from Equation (47) as well as expressions of $T_{g}(0, t)$ and $T_{g}(L, t)$ are shown as follows.

$$
\begin{gathered}
\alpha_{\mathrm{g}, 1}=\rho_{\mathrm{g}, \text { mix. }} \mathrm{C}_{\mathrm{p}, \mathrm{g}, \text { mix. }} ; \alpha_{\mathrm{g}, 2}=\rho_{\mathrm{g}, \text { mix. }} \mathrm{C}_{\mathrm{p}, \mathrm{g}, \text { mix. }} \frac{4 \mathrm{q}_{\mathrm{g}}}{\pi \mathrm{d}_{\mathrm{rz}}^{2}} \\
\alpha_{\mathrm{g}, 3}=\mathrm{h}_{\mathrm{gs}} \frac{\left(1-\varepsilon_{\mathrm{b}}\right)}{\varepsilon_{\mathrm{b}}} \frac{6}{\mathrm{~d}_{\mathrm{P}}} ; \alpha_{\mathrm{g}, 4}=\frac{\rho_{\mathrm{g}, \text { mix. }} \mathrm{C}_{\mathrm{p}, \mathrm{g}, \text { mix. }}}{\lambda_{\mathrm{g}, \text { mix. }}} ; \alpha_{\mathrm{g}, 5}=\frac{\mathrm{h}_{\mathrm{gs}, \text { eff. }}}{\lambda_{\mathrm{g}, \text { eff. }}} \\
\alpha_{\mathrm{g}, 6}=\mathrm{L}\left(6+\mathrm{L} \alpha_{\mathrm{g}, 4}\right)-\frac{\mathrm{L}\left(6+\mathrm{L} \alpha_{\mathrm{g}, 5}\right)\left(1+0.5 \mathrm{~L} \alpha_{\mathrm{g}, 4}\right)}{1+0.5 \mathrm{~L} \alpha_{\mathrm{g}, 5}} \\
\alpha_{\mathrm{g}, 7}(\mathrm{t})=12 \overline{\mathrm{T}}_{\mathrm{g}}(\mathrm{t})+\mathrm{L}^{2}\left(\alpha_{\mathrm{g}, 4} \mathrm{~T}_{\mathrm{g}, \infty}^{\mathrm{Up}}+\alpha_{\mathrm{g}, 5} \mathrm{~T}_{\mathrm{g}, \infty}^{\text {Bot. }}\right)\left[1+\frac{0.5\left(6+\mathrm{L} \alpha_{\mathrm{g}, 5}\right)}{1-0.5 \mathrm{~L} \alpha_{\mathrm{g}, 5}}\right] \\
\mathrm{T}_{\mathrm{g}}(\mathrm{L}, \mathrm{t})=\frac{1}{1-0.5 \mathrm{~L}_{\mathrm{z}} \alpha_{\mathrm{g}, 5}}\left[0.5 \mathrm{~L} \mathrm{~T} \mathrm{~T}_{\mathrm{g}, \infty}\left(\alpha_{\mathrm{g}, 4}+\alpha_{\mathrm{g}, 5}\right)-\left(1+0.5 \mathrm{~L} \alpha_{\mathrm{g}, 4}\right) \frac{\alpha_{\mathrm{g}, 7}(\mathrm{t})}{\alpha_{\mathrm{g}, 6}}\right]
\end{gathered}
$$

Appendix A.3. CIEA Method's Application to the Energy Balance of the Solid Phase in Reaction Zone from PBMR

The boundary conditions (Equations (33) and (34)) of the energy balance for the endothermic reactions at the inlet and outlet of the reaction zone from PBMR were used to transform Equation (30) into Equation (48). The coefficients from Equation (48) as well as expressions of $T_{e r}(0, t)$ and $T_{e r}(L, t)$ are reported as follows.

$$
\begin{array}{r}
\beta_{\mathrm{er}, 1}=\frac{\lambda_{\mathrm{s}, \mathrm{eff}}}{\rho_{\mathrm{s}} \mathrm{C}_{\mathrm{p}, \mathrm{s}}} ; \beta_{\mathrm{er}, 2}=\mathrm{h}_{\mathrm{sg}} \frac{6}{\mathrm{~d}_{\mathrm{p}}} \frac{1}{\rho_{\mathrm{s}} \mathrm{C}_{\mathrm{p}, \mathrm{s}}} \frac{\left(1-\varepsilon_{\mathrm{b}}\right)}{\varepsilon_{\mathrm{b}}} ; \beta_{\mathrm{er}, 3}=\frac{\left(1-\varepsilon_{\mathrm{p}}\right)}{\varepsilon_{\mathrm{p}}} \\
\beta_{\mathrm{er}, 4}=\frac{\mathrm{q}_{\mathrm{h}}}{\lambda_{\mathrm{s}}} ; \beta_{\mathrm{er}, 5}=\frac{\mathrm{h}_{\mathrm{sg}, \text { eff. }}}{\lambda_{\mathrm{s}, \text { eff. }}} ; \beta_{\mathrm{er}, 6}=2 \mathrm{~L}\left[3-\frac{\left(6+\mathrm{L} \beta_{\mathrm{s}, 5}\right)}{2+\mathrm{L} \beta_{\mathrm{s}, 5}}\right] \\
\beta_{\mathrm{er}, 7}(\mathrm{t})=12 \overline{\mathrm{T}}_{\mathrm{er}}(\mathrm{t})-\mathrm{L}^{2}\left(\beta_{\mathrm{er}, 4}-\beta_{\mathrm{er}, 5} \mathrm{~T}_{\mathrm{er}, \infty}^{\text {Bot. }}\right)-\frac{\mathrm{L}^{2}\left(6+\mathrm{L}_{\mathrm{z}} \beta_{\mathrm{er}, 5}\right)\left(\beta_{\mathrm{er}, 4}+\beta_{\mathrm{er}, 5} \mathrm{~T}_{\mathrm{er}, \infty}^{\text {Bot. }}\right)}{2+\mathrm{L} \beta_{\mathrm{er}, 5}} \\
\mathrm{~T}_{\mathrm{er}}(0, \mathrm{t})=\frac{\beta_{\mathrm{er}, 7}(\mathrm{t})}{\beta_{\mathrm{s}, 6}} ; \mathrm{T}_{\mathrm{er}}(\mathrm{L}, \mathrm{t})=\frac{1}{2+\mathrm{L} \beta_{\mathrm{er}, 5}}\left[\mathrm{~L}_{\mathrm{z}}\left(\beta_{\mathrm{er}, 4}+\beta_{\mathrm{er}, 5} \mathrm{~T}_{\mathrm{er}, \infty}^{\text {Bot. }}\right)+2 \frac{\beta_{\mathrm{er}, 7}(\mathrm{t})}{\beta_{\mathrm{er}, 6}}\right]
\end{array}
$$


Appendix A.4. CIEA Method's Application to the Transport of Components $i$ in Reaction Zone from PBMR

The boundary conditions (Equations (37) and (38)) of the mass balance for components i ( $\mathrm{i}=\mathrm{CH}_{4}, \mathrm{H}_{2} \mathrm{O}, \mathrm{CO}$ and $\mathrm{CO}_{2}$ ) at the inlet and outlet of the reaction zone from PBMR have been used to convert Equation (35) into Equation (49). The coefficients from Equation (49) as well as expressions of $F_{i}(0, t)$ and $F_{i}(L, t)$ are described as follows.

$$
\begin{gathered}
\varphi_{\mathrm{f}, 1}=\frac{4 \mathrm{q}_{\mathrm{g}}}{\mathrm{S}_{\mathrm{sp}} \pi \mathrm{d}_{\mathrm{rz}}^{2}} \frac{\mathrm{g}}{\mathrm{u}_{\mathrm{sg}}} ; \varphi_{\mathrm{f}, 2}=\frac{\mathrm{g}}{\mathrm{u}_{\mathrm{sg}}} \frac{\mathrm{D}_{\mathrm{ax}, \mathrm{i}}}{\mathrm{S}_{\mathrm{sp}}} ; \varphi_{\mathrm{f}, 3}=\frac{\mathrm{g}}{\mathrm{u}_{\mathrm{sg}}} \rho_{\mathrm{s}} \mathrm{r}_{\mathrm{rz}}^{2} \mathrm{~L}\left(1-\varepsilon_{\mathrm{b}}\right) ; \varphi_{\mathrm{f}, 4}=\frac{\mathrm{L}}{\varepsilon_{\mathrm{b}}} \frac{\mathrm{u}_{\mathrm{sg}}}{\mathrm{D}_{\mathrm{ax}, \mathrm{i}}} \\
\varphi_{\mathrm{f}, 5}=\frac{\mathrm{L}}{\varepsilon_{\mathrm{b}}} \frac{\mathrm{k}_{\mathrm{sg}}}{\mathrm{D}_{\mathrm{ax}, \mathrm{i}}} ; \varphi_{\mathrm{f}, 6}=\mathrm{L}\left(6+\mathrm{L} \varphi_{\mathrm{f}, 4}\right)-\frac{\mathrm{L}\left(6-\mathrm{L} \varphi_{\mathrm{f}, 5}\right)}{2-\mathrm{L} \varphi_{\mathrm{f}, 5}}\left(2+\mathrm{L} \varphi_{\mathrm{f}, 4}\right) \\
\varphi_{\mathrm{f}, 7}(\mathrm{t})=12 \overline{\mathrm{F}}_{\mathrm{i}}(\mathrm{t})-\mathrm{L}^{2}\left(\varphi_{\mathrm{f}, 5} \mathrm{~F}_{\mathrm{i}, \infty}^{\mathrm{Up}}-\varphi_{\mathrm{f}, 4} \mathrm{~F}_{\mathrm{i}, \infty}^{\text {Bot. }}\right)-\frac{\mathrm{L}^{2}\left(6-\mathrm{L} \varphi_{\mathrm{f}, 5}\right)\left(\varphi_{\mathrm{f}, 4} \mathrm{~F}_{\mathrm{i}, \infty}^{\mathrm{Up} .}+\varphi_{\mathrm{f}, 5} \mathrm{~F}_{\mathrm{i}, \infty}^{\text {Bot. }}\right)}{2-\mathrm{L} \varphi_{\mathrm{f}, 5}} \\
\mathrm{~F}_{\mathrm{i}}(0, \mathrm{t})=\frac{\varphi_{\mathrm{f}, 7}(\mathrm{t})}{\varphi_{\mathrm{f}, 6}} \\
\mathrm{~F}_{\mathrm{i}}(\mathrm{L}, \mathrm{t})=\frac{1}{2-\mathrm{L} \varphi_{\mathrm{f}, 5}}\left[\left(2+\mathrm{L} \varphi_{\mathrm{f}, 4}\right) \frac{\varphi_{\mathrm{f}, 7}(\mathrm{t})}{\varphi_{\mathrm{f}, 6}}-\mathrm{L}\left(\varphi_{\mathrm{f}, 4} \mathrm{~F}_{\mathrm{i}, \infty}^{\mathrm{Up} .}+\varphi_{\mathrm{f}, 5} \mathrm{~F}_{\mathrm{i}, \infty}^{\text {Bot. }}\right)\right]
\end{gathered}
$$

Appendix A.5. CIEA Method's Application to the Transport of $\mathrm{H}_{2}$ in Reaction Zone from PBMR

The boundary conditions (Equations (41) and (42)) of the mass balance of $\mathrm{H}_{2}$ at the inlet and outlet of the reaction zone from PBMR were used to convert Equation (39) into Equation (50). The coefficients from Equation (50) as well as expressions of $\mathrm{F}_{\mathrm{H}_{2}}(0, \mathrm{t})$ and $\mathrm{F}_{\mathrm{H}_{2}}(\mathrm{~L}, \mathrm{t})$ are depicted as follows.

$$
\begin{gathered}
\vartheta_{\mathrm{H}_{2}, 1}=\frac{\mathrm{g}}{\mathrm{u}_{\mathrm{sg}}} \frac{4 \mathrm{q}_{\mathrm{g}}}{\mathrm{S}_{\mathrm{sp}} \pi \mathrm{d}_{\mathrm{rz}}^{2}} ; \vartheta_{\mathrm{H}_{2}, 2}=\frac{\mathrm{g}}{\mathrm{u}_{\mathrm{sg}}} \frac{\mathrm{D}_{\mathrm{ax}, \mathrm{H}_{2}}}{\mathrm{~S}_{\mathrm{sp}}} ; \vartheta_{\mathrm{H}_{2,3}}=\frac{\mathrm{g}}{\mathrm{u}_{\mathrm{sg}}} \rho_{\mathrm{s}} \mathrm{r}_{\mathrm{rs}}^{2} \mathrm{~L}\left(1-\varepsilon_{\mathrm{b}}\right) \\
\vartheta_{\mathrm{H}_{2}, 4}=\frac{\mathrm{g}}{\mathrm{u}_{\mathrm{sg}}} \pi \mathrm{d}_{\mathrm{rs}}^{2} \mathrm{~J}_{\mathrm{H}_{2}, \mathrm{per} .} ; \vartheta_{\mathrm{H}_{2}, 5}=\frac{\mathrm{L}}{\varepsilon_{\mathrm{b}}} \frac{\mathrm{u}_{\mathrm{sg}}}{\mathrm{D}_{\mathrm{ax}, \mathrm{H}_{2}}} ; \vartheta_{\mathrm{H}_{2}, 6}=\frac{\mathrm{L}}{\varepsilon_{\mathrm{b}}} \frac{\mathrm{k}_{\mathrm{sg}, \mathrm{eff} .}}{\mathrm{D}_{\mathrm{ax}, \mathrm{H}_{2}}} \\
\vartheta_{\mathrm{H}_{2}, 7}=\mathrm{L}\left(6+\mathrm{L} \vartheta_{\mathrm{H}_{2}, 5}\right)-\frac{\mathrm{L}\left(6-\mathrm{L} \vartheta_{\mathrm{H}_{2}, 6}\right)}{2-\mathrm{L} \vartheta_{\mathrm{H}_{2}, 6}}\left(2+\mathrm{L} \vartheta_{\mathrm{H}_{2}, 5}\right) \\
\vartheta_{\mathrm{H}_{2}, 8}(\mathrm{t})=12 \overline{\mathrm{F}}_{\mathrm{H}_{2}}(\mathrm{t})-\mathrm{L}^{2}\left(\vartheta_{\mathrm{H}_{2}, 6} \mathrm{~F}_{\mathrm{H}_{2}, \infty}^{\mathrm{Bot}}-\vartheta_{\mathrm{H}_{2,5}} \mathrm{~F}_{\mathrm{H}_{2}, \infty}^{\mathrm{Up}}\right)-\frac{\mathrm{L}\left(6-\mathrm{L} \vartheta_{\mathrm{H}_{2}, 6}\right)}{2-\mathrm{L} \vartheta_{\mathrm{H}_{2}, 6}} \mathrm{~L} \\
\left(\vartheta_{\mathrm{H}_{2,}, 5} \mathrm{~F}_{\mathrm{H}_{2}, \infty}^{\mathrm{Up}_{2}}+\vartheta_{\mathrm{H}_{2}, 6} \mathrm{~F}_{\mathrm{H}_{2}, \infty}^{\mathrm{Bot}}\right) \\
\mathrm{F}_{\mathrm{H}_{2}}(\mathrm{~L}, \mathrm{t})=\frac{1}{2-\mathrm{L} \vartheta_{\mathrm{H}_{2}, 6}}\left[\frac{(2, \mathrm{t})=\frac{\vartheta_{\mathrm{H}_{2}, 8}(\mathrm{t})}{\vartheta_{\mathrm{H}_{2}, 7}}}{\vartheta_{\mathrm{H}_{2}, 7}}\right.
\end{gathered}
$$

\section{References}

1. Murmura, M.A.; Cerbelli, S.; Annesini, M.C. Modeling fixed bed membrane reactors for hydrogen production through steam reforming reactions: A critical analysis. Membranes 2018, 8, 34. [CrossRef] [PubMed]

2. Li, Q.; Lv, Y.; Ding, R.; Wang, L.; Ren, N.; Wei, L.; Li, Y. Hydrogen production efficiency and microbial community of ethanol-type fermentation. J. Renew. Sustain. Energy 2019, 11, 013105. [CrossRef]

3. Cruz, B.M.; Silva, J.D. A two-dimensional mathematical model for the catalytic steam reforming of methane in both conventional fixed-bed and fixed-bed membrane reformers for the production of hydrogen. Int. J. Hydrog. Energy 2017, 42, 23670-23690. [CrossRef]

4. Lima, K.P.M.; Dias, V.F.; Silva, J.D. Numerical modelling for the solar driven bi-reforming of methane for the production of syngas in a solar thermochemical micro-packed bed reformer. Int. J. Hydrog. Energy 2020, 45, 10353-10369. [CrossRef]

5. Anjos, E.B.; Filho, A.M.S.; Silva, J.D. Numerical simulation of the steam reforming of toluene in a fixed-bed catalytic reformer to produce hydrogen. J. Braz. Soc. Mech. Sci. Eng. 2020, 42, 114. [CrossRef]

6. Silva, J.D.; Abreu, C.A.M. Modelling and simulation in conventional fixed-bed and fixed-bed membrane reformers for the steam reforming of methane. Int. J. Hydrog. Energy 2016, 41, 11669-11674. [CrossRef]

7. Dias, V.F.; Silva, J.D. Mathematical modelling of the solar-Driven steam reforming of methanol for a solar thermochemical micro-Fluidized bed reformer: Thermal performance and thermochemical conversion. Braz. Soc. Mech. Sci. Eng. 2020, $42,447$. [CrossRef] 
8. Sakbodin, M.; Schulman, E.; Oh, S.C.; Pan, Y.; Wachsman, E.D.; Liu, D. Dual utilization of greenhouse gases to produce C $2+$ hydrocarbons and syngas in a hydrogen-permeable membrane reformer. J. Membr. Sci. 2020, 595, 117557. [CrossRef]

9. Durán, P.; Sanz-Martínez, A.; Soler, J.; Menéndez, M.; Herguido, J. Pure hydrogen from biogas: Intensified methane dry reforming in a two-zone fluidized bed reformer using permselective membranes. Chem. Eng. J. 2019, 370, 772-781. [CrossRef]

10. Yang, X.; Wang, S.; Hu, B.; Zhang, K.; He, Y. Estimation of concentration polarization in a fluidized bed reformer with Pd-based membranes via CFD approach. J. Membr. Sci. 2019, 581, 262-269. [CrossRef]

11. Barba, D.; Capocelli, M.; De Falco, M.; Franchi, G.; Piemonte, V. Mass transfer coefficient in multi-stage reformer/membrane modules for hydrogen production. Membranes 2018, 8, 109. [CrossRef] [PubMed]

12. Jamalabadi, M.Y.A. Numerical simulation of carbon dioxide sorption circulating fluidized bed used in solid oxide fuel cell. J. Therm. Anal. Calorim. 2020, 139, 2565-2575. [CrossRef]

13. Cruellas, A.; Ververs, W.; Annaland, M.V.S.; Gallucci, F. Experimental investigation of the oxidative coupling of methane in a porous membrane reactor: Relevance of back-permeation. Membranes 2020, 10, 152. [CrossRef]

14. Hussain, A.; Seidel-Morgenstern, A.; Tsotsas, E. Heat and mass transfer in tubular ceramic membranes for membrane reformers. Int. J. Heat Mass Transf. 2006, 49, 2239-2253. [CrossRef]

15. Silva, J.D. Numerical modelling for a catalytic trickle-bed reformer using Laplace transform technique. Chem. Eng. Trans. 2015, 43, 1573-1578. [CrossRef]

16. Silva, J.D.; Abreu, C.A.M. Mathematical modeling of a three-phase trickle bed reformer. Braz. J. Chem. Eng. 2012, 29, 567-576. [CrossRef]

17. Iulianelli, A.; Ghasemzadeh, K.; Basile, A. Progress in methanol steam reforming modelling via membrane reactors technology. Membranes 2018, 8, 65. [CrossRef]

18. Zambrano, D.; Soler, J.; Herguido, J.; Menéndez, M. Conventional and improved fluidized bed reformers for dry reforming of methane: Mathematical models. Chem. Eng. J. 2020, 393, 124775. [CrossRef]

19. Jowkary, H.; Farsi, M.; Rahimpour, M.R. Supporting the propane dehydrogenation reformers by hydrogen permselective membrane modules to produce ultra-pure hydrogen and increasing propane conversion: Process modeling and optimization. Int. J. Hydrog. Energy 2020, 45, 7364-7373. [CrossRef]

20. Dittrich, C.J. The role of heat transfer on the feasibility of a packed-bed membrane reformer for propane dehydrogenation. Chem. Eng. J. 2020, 381, 122492. [CrossRef]

21. Gerardine, B.G.; Ritter, J.A.; White, R.E. Comparison of finite difference and control volume methods for solving differential equations. Comp. Chem. Eng. 2000, 24, 2633-2654. [CrossRef]

22. Silva, J.D.; Oliveira, C.B. Fluidynamics modelling for a fixed bed gasifier using Laplace transform finite difference method. Proc. Eng. 2012, 42, 753-769. [CrossRef]

23. Chompupun, T.; Limtrakul, S.; Vatanatham, T.; Kanhari, C.; Ramachandran, P.A. Experiments, modeling and scaling-up of membrane reformers for hydrogen production via steam methane reforming. Chem. Eng. Process.-Process Intensif. 2018, 134, 124-140. [CrossRef]

24. Lee, H.; Kim, A.; Lee, B.; Lim, H. Comparative numerical analysis for an efficient hydrogen production via a steam methane reforming with a packed-bed reformer, a membrane reformer, and a sorption-enhanced membrane reformer. Energy Convers. Manag. 2020, 213, 112839. [CrossRef] 